\title{
Investigación española en las áreas de adicciones y de trastornos de la alimentación: análisis de la producción, colaboración e impacto científico (1962-2017)
}

\author{
Rafael Aleixandre-Benavent*, Víctor Agulló-Calatayud**, Adolfo Alonso-Arroyo***, \\ Francisco Jesús Bueno-Cañigral****, Lourdes Castelló-Cogollos**, Rut Lucas-Domínguez***, \\ David Melero-Fuentes*****, Andrea Sixto-Costoya***, Antonio Vidal-Infer***, Juan Carlos Valderrama-Zurián*** \\ *Unidad de Investigación e Información Social y Sanitaria-UISYS (CSIC-Universitat de València). \\ Ingenio (CSIC-Universitat Politècnica de València). \\ Correo-e: Rafael.Aleixandre@uv.es | ORCID iD: http://orcid.org/0000-0002-6678-8844 \\ **Unidad de Investigación e Información Social y Sanitaria-UISYS (CSIC-Universitat de València). \\ Departamento de Sociología y Antropología Social. Universitat de València. \\ Correo-e: Victor.Agullo@uv.es | ORCID iD: http://orcid.org/0000-0002-0720-1572 \\ Correo-e: Lourdes.Castello@uv.es | ORCID iD: http://orcid.org/0000-0002-0305-3154 \\ ***Unidad de Investigación e Información Social y Sanitaria-UISYS (CSIC-Universitat de València). \\ Departamento de Historia de la Ciencia y Documentación. Universitat de València. \\ Correo-e: Adolfo.Alonso@uv.es | ORCID iD: http://orcid.org/0000-0002-5084-2818 \\ Correo-e: Rut.Lucas@uv.es | ORCID iD: http://orcid.org/0000-0002-4697-7832 \\ Correo-e: Andrea.Sixto@uv.es | ORCID iD: http://orcid.org/0000-0001-9162-8992 \\ Correo-e: Antonio.Vidal-Infer@uv.es | ORCID iD: http://orcid.org/0000-0002-7860-8652 \\ Correo-e: Juan.Valderrama@uv.es | ORCID iD: http://orcid.org/0000-0001-5787-6853 \\ ****Servicio de Drogodependencias (PMD/UPCCA-València). Concejalía de Salud y Consumo. Ajuntament de València. \\ Correo-e: fjbueno@valencia.es | ORCID iD: http://orcid.org/0000-0003-0168-6035 \\ *****Instituto de Documentación y Tecnologías de la Información (INDOTEI). Universidad Católica de Valencia "San Vicente Mártir" \\ Correo-e: David.Melero@ucv.es | ORCID iD: http://orcid.org/0000-0002-4610-3000
}

Cómo citar este artículo/Citation: Aleixandre-Benavent, R., Agulló-Calatayud, V., Alonso-Arroyo, A., Bueno-Cañigra, F. J., Castelló-Cogollos, L., Lucas-Domínguez, R., Melero-Fuentes, D., Sixto-Costoya, S., Vidal-Infer, A., Valderrama-Zurián, J. C. (2021). Investigación española en las áreas de adicciones y de trastornos de la alimentación: análisis de la producción, colaboración e impacto científico (1962-2017). Revista Española de Documentación Científica, 44 (3), e299. https://doi.org/10.3989/ redc.2021.3.1766

Resumen: Las adicciones y los trastornos de la alimentación constituyen uno de los principales problemas de salud en la sociedad contemporánea al que se destinan gran cantidad de recursos destinados a la investigación para su prevención y tratamiento. Objetivos. Analizar la investigación española en el área de adicciones y trastornos de la alimentación desde que se publicó el primer trabajo recogido en bases de datos bibliográficas y desde una perspectiva bibliométrica. Metodología. Para obtener la información necesaria se consultaron las bases de datos Web of Science, Scopus y bases de datos del CSIC. Se elaboró un perfil de búsqueda específico. Se obtuvieron indicadores bibliométricos de producción, colaboración e impacto científico. Se utilizó el análisis de redes sociales para identificar las redes de investigadores y sus relaciones de colaboración, así como la red de co-palabras clave. Resultados. La producción científica aumentó progresivamente durante los casi 50 años analizados. Los trabajos se publicaron en una gran variedad de revistas nacionales y extranjeras, entre las que destacaron Adicciones, Revista Española de Drogodependencias, Trastornos Adictivos y Salud y Drogas. Conclusiones. La producción, colaboración e impacto de la investigación ha sido creciente y las adicciones más estudiadas fueron el tabaquismo, alcoholismo, consumo de cocaína y consumo de anfetaminas. 
Palabras clave: evaluación bibliométrica; adicciones; trastornos de la alimentación; producción científica; redes de colaboración; redes de co-palabras clave; impacto científico

\title{
Spanish research in the areas of addictions and eating disorders: analysis of scientific production, collaboration and impact (1962-2017)
}

\begin{abstract}
Eating disorders and addictions are one of the major health problems in contemporary society and a great deal of resources are devoted to research into their prevention and treatment. Objectives. To analyze Spanish research in the area of food addictions and disorders since the first work collected in bibliographic databases was published and from a bibliometric perspective. Methodology. To obtain the necessary information, the Web of Science, Scopus and CSIC databases were consulted. A specific search profile was drawn up. Bibliometric indicators of production, collaboration and scientific impact were obtained. The analysis of social networks was used to identify the groups of researchers and their collaborative relationships, as well as the network of key co-words. Results. Scientific production increased progressively during the almost 50 years analyzed. The papers were published in a wide diversity of national and foreign journals, among which are: Addictions, Revista Española de Drogodependencias, Trastornos Adictivos and Salud y Drogas stand out. Conclusions. The production, collaboration and impact of the research increased and the most studied addictions were smoking, alcoholism, cocaine use and amphetamine use.
\end{abstract}

Keywords: bibliometric assessment; addictions; eating disorders; scientific production; collaborative networks; co-word networks; scientific impact.

Copyright: (c) 2021 CSIC. Este es un artículo de acceso abierto distribuido bajo los términos de la licencia de uso y distribución Creative Commons Reconocimiento 4.0 Internacional (CC BY 4.0).

\section{INTRODUCCIÓN}

Desde que a finales de la década de los 70 se extendió el consumo de drogas de abuso, los gobiernos de muchos países y las organizaciones de ámbito internacional han ido creando organismos dedicados a la investigación de todos los aspectos relacionados con este problema (De Miguel, 1994; PNSD, 1997; Torres Hernández y otros, 2008).

En España se fundó, en 1985, el Plan Nacional Sobre Drogas (PNSD) con el fin de potenciar y coordinar las políticas de drogodependencias de las administraciones públicas. En su declaración de principios, el PNSD recalcaba que era necesario potenciar la investigación permanente tanto en lo que se refiere al conocimiento de la realidad, como en lo que corresponde a investigaciones aplicadas, que permitan delimitar nuevas experiencias de prevención y tratamiento. Llamaba la atención sobre la necesidad de desarrollar métodos normalizados que hicieran posible comparar y completar los resultados obtenidos en diferentes trabajos y en diferentes épocas (Plan Nacional Sobre Drogas, 2020). También se promovía la colaboración con otros organismos internacionales, como el National Institute on Drug Abuse (NIDA) de los Estados Unidos (National Institute on Drug Abuse, 2020) y el Observatorio Europeo de las Drogas y las Toxicomanías, creado para proporcionar a la UE y a sus Estados miembros los datos que necesitaran para elaborar leyes y estrategias de drogas bien fundadas, así como para ayudar a los profesionales que trabajan en este campo a identificar las mejores prácticas y las nuevas áreas de investigación (EMCDDA, 2020).
Posteriormente, en el ámbito de las adicciones se han incluido otras adicciones no químicas como el juego patológico, que el Manual diagnóstico y estadístico de los trastornos mentales-DSM-V (APA, 2013) lo incluye en el capítulo de Trastornos relacionados con Sustancias y otras Adicciones. El motivo es que participan sistemas de recompensa similares a las adicciones químicas y originan síntomas conductuales similares a las adicciones a sustancias (Carbonell, 2013).

Un grupo de trastornos que comparte similitudes con las adicciones por su repercusiones en la salud pública es el de los trastornos de la alimentación (Carta y otros, 2014; Mond, 2016; Moessner y Baurer, 2017; Hudson y Pope, 2018). Una muestra de su importancia en la literatura científica es que una búsqueda realizada en marzo de 2020 en la base de datos PubMed sobre estos trastornos con el Medical Subject Headings "Feeding and Eating Disorders" obtenía más de treinta mil artículos, con una tasa de publicación de alrededor de 1.300 artículos anuales. Este crecimiento en el número de publicaciones se acompaña del surgimiento de nuevas revistas dedicadas a publicar las investigaciones de estos trastornos (como, por ejemplo, International Journal of Eating Disorders, editada por John Wiley \& Sons).

Se han publicado numerosos trabajos bibliométricos que analizaron diversas sustancias de abuso o aspectos de las adicciones, entre ellos, el alcoholismo (González-Alcaide y otros, 2013; Bramness y otros, 2014; Clifford and Shakeshaft, 2016), tabaquismo (Briganti y otros, 2003; Neff y otros, 2015; 
Li y otros, 2015; Willemsen and Nagelhout, 2016; Metse y otros, 2017; Mahabee-Gittens y otros, 2017; Wang y otros, 2018; Zyoud y otros, 2018; de Granda-Orive y otros, 2018), consumo de cocaína (Moreira y otros, 2015; Zyoud y otros, 2017) y consumo de opioides (Akbar y otros, 2019). Algunos trabajos se abordaron desde una perspectiva nacional (González y otros, 2008) y otros europea (Sánchez-Carbonell y otros, 2005) y mundial (Helinski and Spanagel, 2011; Blobaum, 2013; González y otros, 2015; Khalili y otros, 2018; Tran y otros, 2019). Algunas de las características comunes de la mayor parte de estos estudios son: el crecimiento notable de las publicaciones; el nacimiento y la consolidación de revistas especializadas en conductas adictivas, pero también la publicación en revistas de diferentes especialidades o en revistas de propósito general o multidisciplinar; la naturaleza polifacética del área; el surgimiento de nuevas adicciones, como la adicción al juego, a las nuevas tecnologías y a nuevas drogas de síntesis; la consolidación de las adicciones como área temática en las principales bases de datos bibliográficas; la cada vez mayor colaboración internacional.

Una forma de conocer el estado en el que se encuentra la investigación en adicciones y en los trastornos de la alimentación en España y su evolución es a través del análisis de las publicaciones y la determinación de medidas que la cuantifiquen y midan su impacto. Los indicadores bibliométricos permiten caracterizar el estado de la investigación en un área, identificando sus principales componentes (autores y núcleos de investigadores que componen el frente de investigación e instituciones), las relaciones existentes entre ellos (redes de colaboración, cooperación internacional) y la repercusión e impacto que tienen las investigaciones en los trabajos posteriores (Valderrama-Zurián y otros, 2015; Bramness y otros, 2014). También permiten realizar una aproximación al conocimiento generado en las investigaciones a través del análisis conceptual de las publicaciones (Batman y Miles, 2015; Kushner, 2014).

El objetivo general de este trabajo es analizar, mediante la utilización de indicadores bibliométricos y análisis de redes sociales, la actividad científica de los investigadores en adicciones y en trastornos de la alimentación que trabajan en instituciones españolas. Los objetivos específicos se centran en identificar: la evolución anual de los trabajos publicados; las revistas de publicación y su impacto; los autores e instituciones y sus patrones de producción, colaboración e impacto; los trabajos más citados y sus características; los núcleos de autores e instituciones que conforman las redes de colaboración; las palabras clave más frecuentes y la red de cowords que permita disponer de una mapa conceptual de la investigación en el campo. La finalidad es que la información obtenida sirva como instrumento para el conocimiento de las fortalezas y debilidades de las dos áreas y disponer de datos que contribuyan a facilitar la adopción de decisiones informadas sobre la política científica.

\section{MATERIAL Y MÉTODOS}

Siguiendo la metodología de otros estudios (Valderrama y otros, 1997; Álvarez y Aleixandre 2001, Valderrama y otros, 2017), y para poder disponer de forma exhaustiva de la información científica sobre adicciones, tanto a sustancias químicas como comportamentales, y sobre trastornos de la alimentación se han utilizado las bases de datos bibliográficas: Science Citation Index Expanded (SCIE) y Social Science Citation Index (SSCI) incluídas en Web of Science Core Collection (WoS) de Clarivate Analytics, Scopus de Elsevier, que incluye Medline; Bases de datos del Consejo Superior de Investigaciones Científicas-CSIC (hoy en día accesibles a través de "Índices CSIC"). Estas bases de datos son las habitualmente utilizadas en los estudios bibliométricos, pues incluyen las revistas de mayor importancia y calidad a nivel nacional e internacional, y han sido empleadas ampliamente por numerosos investigadores (Arbinaga, 2002; Granda-Orive, 2009; Villanueva y otros, 2007; Bramness y otros, 2014; Li y otros, 2015).

Para la identificación de los documentos objeto de estudio se realizó una búsqueda en las bases de datos mencionadas de todos los trabajos sobre adicciones y sobre trastornos de la alimentación en los que hubiera intervenido al menos una institución española. La estrategia de consulta tuvo en cuenta tanto la sustancia psicoactiva (como "cocaína" o "cannabis") como los términos que se refieren a sustancias psicoactivas en sentido amplio (como "droga", "drogodependencia" y "abuso de sustancias), la adicción al juego y a las nuevas tecnologías, y los trastornos de la conducta alimentaria, como la anorexia, la vigorexia y la bulimia, ya que existen aspectos clínicos cognitivos y conductuales comunes entre los trastornos de la alimentación, la adicción al juego y a las nuevas tecnologías y las conductas adictivas. Con los resultados obtenidos, se realizó un análisis conceptual de los registros para valorar la pertinencia y calidad de la búsqueda. Este procedimiento fue efectuado mediante la lectura de los resúmenes y palabras clave de los artículos obtenidos. Esta lectura ha permitido obtener una lista ampliada que recoge toda la terminología utilizada en los trabajos sobre adicciones o sobre trastornos de la alimentación. El 
perfil de búsqueda utilizado se adaptó a cada una de las bases de datos. La limitación temporal se estableció hasta 2017 y los tipos de documentos considerados fueron artículos de investigación (habitualmente indexados como articles) y revisiones (reviews). Las búsquedas se realizaron la primera semana de junio de 2018.

Todos los registros se exportaron a una base de datos en Access de Microsoft utilizando un programa de desarrollo propio denominado Bibliométricos. Los registros bibliográficos duplicados entre Scopus, WoS y bases de datos del CSIC se integraron en una único registro bibliográfico utilizando el algoritmo de Valderrama y otros, (2015). El número de registros resultantes fue de 16.442 , de los que 9.241 registros procedían de WoS, 2.671 de Scopus y 4.530 de las Bases de datos del CSIC.

A continuación se unificaron las diferentes variantes de los autores e instituciones comprobando la coincidencia en los lugares de trabajo de las diferentes variantes. En el caso de las instituciones, además se separaron las firmas de los registros en los que aparecía más de una única adscripción institucional.

Se calcularon diversas medidas o indicadores de productividad científica, colaboración e impacto, tanto del grupo de trabajos sobre adicciones como del de trastornos de la alimentación. El principal indicador de producción es el número de trabajos publicados, que se ha aplicado a los autores, instituciones, revistas y áreas temáticas. El indicador de colaboración determinado ha sido el índice de colaboración (o índice de firmantes por trabajo). Se elaboraron redes de autores, instituciones y países. Para la llevar a cabo la representación gráfica se utilizó el software Pajek, que integra el algoritmo de poda Pathfinder para reducir el espacio dimensional y el algoritmo Kamada-Kawai como algoritmo de visualización. La red de colaboración entre autores se ha elaborado aplicando un umbral de trece o más artículos publicados en colaboración. Con este umbral se constituyeron 72 redes de coautores integrados por 470 investigadores. Se eligió este umbral porque con él se facilitaba la visualización de las redes, mientras que si se disminuía, el número de integrantes de cada red se enmascaraba su correcta visualización. Con este umbral se conformó una red de 90 autores, otra de 80 , dos de 11 autores, 5 redes de 10 y 63 redes con menos de 10 autores. Para la construcción de la red de colaboración entre instituciones se aplicó un umbral de 40 colaboraciones o más en común, ya que con umbrales más bajos el número de instituciones que resultaban impedía su visualización. La red de colaboración entre países se confeccionó estableciendo como umbral la existencia de más de 40 colaboraciones en común. Esta red se ha mostrado en forma de mapa geográfico mundial. En las redes resultantes, el diámetro de las esferas es proporcional al número de autores o instituciones distintas con los que colabora cada nodo (autores, instituciones o países). El grosor de las líneas es proporcional al número de veces que dos investigadores o instituciones "conectadas" colaboran en los artículos.

Como indicadores de impacto se determinó el número de citas en Web of Science y en Scopus, el número medio de citas por trabajo, el factor de impacto de 5 años de las revistas, el cuartil que ocupaban en Journal Citation Reports (edición de 2017) y los trabajos altamente citados en SCIE y SSCI. Se ha utilizado el factor de impacto de las revistas en Journal Citation Report porque se utiliza habitualmente en los estudios bibliométricos como indicador de la calidad de las revistas (Rizkallah, Sin, 2010). Cabe señalar que el factor de impacto se basa en el número de citas brutas y que no puede compararse entre las distintas disciplinas, pero sí sus respectivas posiciones en la clasificación de su categoría temática o cuartil (Leydesdorff, Bornmann, 2011; Dorta-González y Dorta-Gonzalez, 2013).

El análisis de las palabras clave se llevó a cabo a partir de los 10.934 registros que incluían este campo cumplimentado, lo que supone el $66,5 \%$ de los trabajos. La normalización de estas palabras consistió fundamentalmente en la agrupación de sinónimos (como "cannabis" y "marihuana" o "abstinence syndrome" y "withdrawal syndrome"), el desglose de siglas ("THC" y "tetrahidrocannabinol" o "Mdma" y "3,4 Methylenedioxymethamphetamine"), agrupación de singulares y plurales (amphetamine" y "amphetamines") y agrupación de palabras compuestas con guión y sin guión ("nutritional-status" y "nutritional status"). Esta normalización fue realizada por médicos, psicólogos, sociólogos y documentalistas con experiencia en los trastornos que se estudian.

Para el análisis temático y de ámbitos del conocimiento se ha utilizado una combinación de indicadores bibliométricos (frecuencia y evolución de las palabras clave, y áreas temáticas en las que se clasifican las revistas) y técnicas de visualización de co-palabras (coincidencia de parejas de palabras en varios artículos) basadas en el análisis de redes sociales mediante mapas etiquetados en los que cada nodo corresponderá a una palabra clave. En esta red, el tamaño de los nodos es proporcional a su valor o frecuencia. 


\section{RESULTADOS}

\subsection{Evolución anual de los artículos publica- dos y de las revistas}

Desde 1962, año en el que se ha identificado en las bases de datos consultadas el primer artículo publicado, hasta 2017, último año analizado, se publicaron 16.442 artículos. El número de artículos ha aumentado progresivamente desde el periodo 1962-1972, en el que se publicaron 27 trabajos, hasta el quinquenio 2013-2017, en el que se publicaron 3.779. El mayor número de citas corresponde al quinquenio 2008-2012, tanto en WoS como en Scopus. Del total de trabajos, $15.211(92,5 \%)$ correspondían a adicciones y $1.553(9,4 \%)$ a trastornos de la alimentación. La suma de ambos porcentajes supera el $100 \%$ debido a los trabajos que trataban simultáneamente de ambas temáticas. La figura 1 permite observar gráficamente la evolución quinquenal de los trabajos publicados en cada una de las dos áreas.

Figura 1. Evolución de los artículos publicados por quinquenios

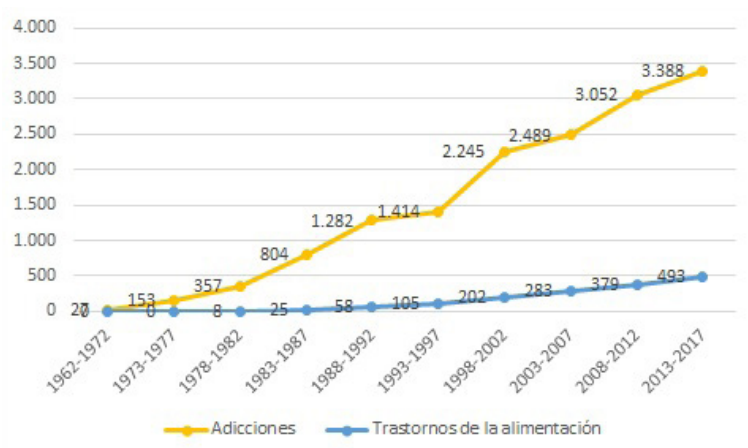

Los trabajos se divulgaron en 2.161 revistas diferentes. La tabla I muestra las 29 revistas que publicaron más de 80 artículos. Las revistas que incluyeron un mayor número de artículos en las dos grandes áreas analizadas fueron Adicciones $(n=900)$, seguida de Medicina Clínica $(n=617)$, Revista Española de Drogodependencias $(n=586)$ y Trastornos Adictivos $(n=358)$. Estas revistas son las que publicaron también un mayor número de trabajos sobre adicciones. Las que publicaron más trabajos sobre trastornos de la alimentación fueron European Eating Disorders Review (93), Nutrición Hospitalaria (63) y Actas Españolas de Psiquiatría (57).

De estas 29 revistas, 19 se editaban en España, cuatro en Reino Unido, dos en Estados Unidos, una en Alemania y otra en Suiza. Las revistas extranjeras con un mayor número de trabajos en las dos áreas fueron Drug and Alcohol Dependence $(n=179)$, Alcoholism-Clinical and Experimental Research $(n=168)$ y Alcohol and Alcoholism $(n=155)$. La revista con mayor factor de impacto ha sido Addiction ( $\mathrm{FI}=5,953$ ), seguida de Addiction Biology $(\mathrm{FI}=5,578)$ y Drug and Alcohol Dependence $(\mathrm{FI}=3,322)$. Estas revistas son también las que tienen un mayor factor de impacto de 5 años. Por número de citas en WoS destacan, en el área de las adicciones, Drug and Alcohol Dependence $(n=4.388)$, Alcoholism-clinical and Experimental Research $(\mathrm{n}=4.175)$ y Alcohol and Alcoholism $(\mathrm{n}=3.516)$. En trastornos de la alimentación, el mayor número de citas en WoS corresponde a European Eating Disorders Review (1.128), Nutrición Hospitalaria (258) y Actas Españolas de Psiquiatría (180). En Scopus, Alcohol and Alcoholism $(\mathrm{n}=4.440)$ sobrepasa a Alcoholism-clinical and Experimental Research (4.231). Las revistas españolas más citadas han sido Adicciones (1.452 en WoS y 2.836 en Scopus) y Medicina Clínica (1.896 en WoS y 2.085 en Scopus). En trastornos de la alimentación, el mayor número de citas en Scopus lo han obtenido European Eating Disorders Review (1.330), Nutrición Hospitalaria (463) y Actas Españolas de Psiquiatría (329) y Plos One (226). Algunas revistas de la tabla carecen de citas y de factor de impacto porque no se encuentran incluidas en la base de datos WoS. Es el caso de Revista Española de Drogodependencias, la tercera revista en la que se han publicado un mayor número de artículos, Trastornos Adictivos (la cuarta revista más productiva), Comunidad y Drogas, Drogalcohol y Health and Addictions / Salud y Drogas, entre otras. En la figura 2 se puede seguir la evolución quinquenal de las publicaciones según los cuartiles de las revistas en JCR. Como puede apreciarse, en el último quinquenio (2013-2017) los trabajos en revistas del primer cuartil superan a los del segundo cuartil, y en los dos últimos quinquenios (2008-2017) los trabajos en revistas del segundo cuartil superan a los del tercero. Las revistas españolas más productivas suelen situarse en los cuartiles tercero y cuarto del JCR, con la excepción de Archivos de Bronconumología y de Psicothema que se clasifican en el segundo cuartil, mientras que las extranjeras predominan en el primer cuartil, como Drug and Alcohol Dependence, Addiction, Addiction Biology, Plos One y European Eating Disorders Review. El porcentaje total de trabajos publicados en revistas indexadas en JCR ha sido del $66,5 \%$. Este porcentaje ha aumentado progresivamente a lo largo del periodo, de manera que ha pasado del $40,6 \%$ en los dos quinquenios que abracan el periodo $1983-$ 1992 , al $70,4 \%$ en el quinquenio $2003-2007$ y al $80,5 \%$ en el quinquenio 2013-2017. 


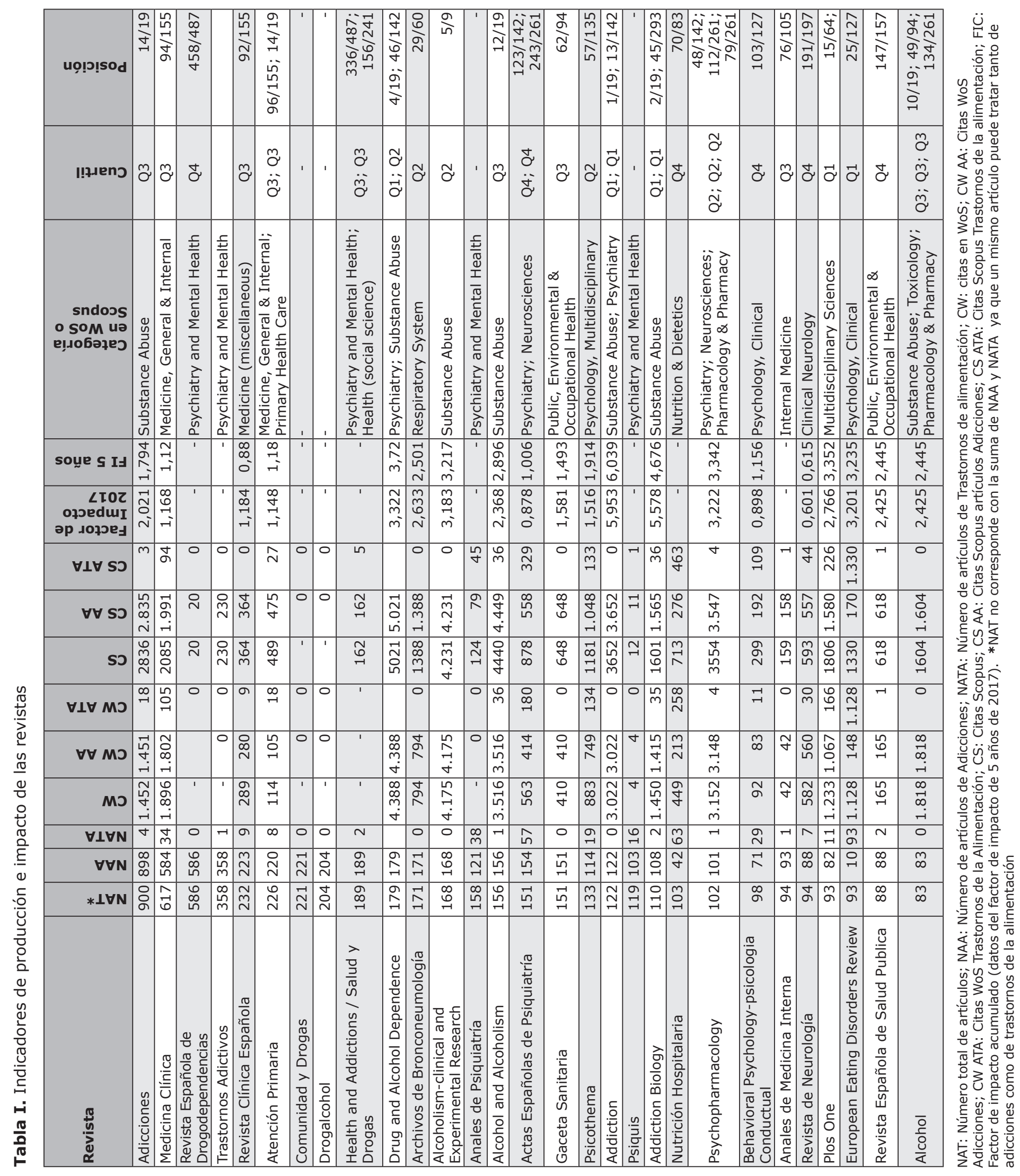


Figura 2. Evolución diacrónica por quinquenios de las publicaciones según los cuartiles de las revistas

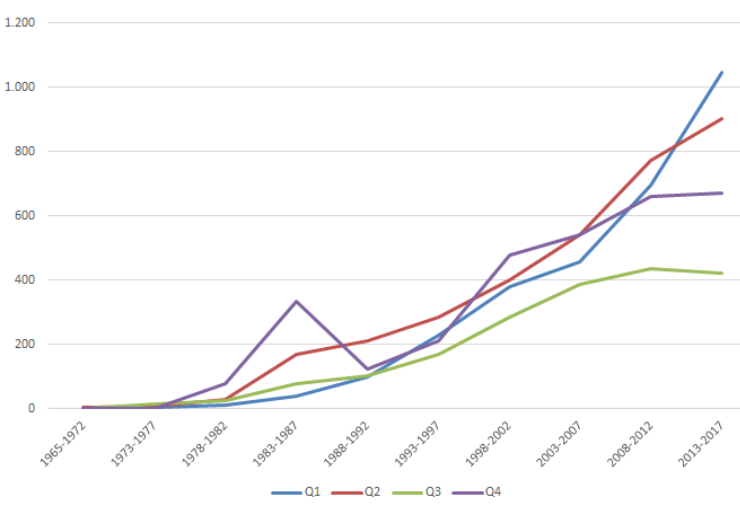

\subsection{Productividad e impacto de los autores}

El número de autores diferentes que han publicado los artículos ha sido de 38.237. Casi tres cuartas partes de los autores $(70,5 \%)$ han publicado un único artículo, el $13 \%$ han publicado dos artículos, el 5,3\% han publicado tres y el 2,9\% han publicado cuatro. El porcentaje de autores que han publicado más de cuatro artículos es, en conjunto, de un $8,3 \%$ (tabla II).

La tabla III presenta varios indicadores sobre los autores más productivos, tanto en valores totales como desglosados en las dos grandes áreas analizadas: el número y porcentaje de artículos publicados en revistas españolas y extranjeras, el número de citas recibidas, la media de citas por artículo y el factor de impacto acumulado, que es el resultado de multiplicar el factor de impacto de cada revista por el número de artículos publicados en la misma. La observación conjunta de varios indicadores nos permite disponer de una dimensión más completa de cada investigador. Los autores más productivos en el área de las adicciones (con más de 150 artículos) Becoña Iglesias, Departamento de Psicología Clínica y Psicobiología de la Universidade de Santiago de Compostela $(n=192)$, Gual Solé, Unitat de Alcohología de la Generalitat de Catalunya, Barcelona $(n=172)$, y de la Torre, $(n=153)$, del grupo de Farmacología Integrada y Neurociencia de Sistemas (cuyos investigadores están integrados en las universidades Pompeu Fabra y Autònoma de Barcelona). Los autores más productivos en el área de los trastornos de la alimentación han sido Fernández Aranda, responsable de la Unidad de Trastornos de la Alimentación del Servicio de Psiquiatría del Hospital Universitario de Bellvitge (Hospitalet de Llobregat, Barcelona) $(n=147)$, Jiménez Murcia (80) y Granero Pérez (65).
Tabla II. Número de autores con $\mathrm{n}$ artículos publicados

\begin{tabular}{|c|c|c|}
\hline No de artículos & No de autores & $\%$ \\
\hline 1 & 26.970 & $70,5 \%$ \\
\hline 2 & 4.967 & $13,0 \%$ \\
\hline 3 & 2.039 & $5,3 \%$ \\
\hline 4 & 1.092 & $2,9 \%$ \\
\hline 5 & 720 & $1,9 \%$ \\
\hline 6 & 515 & $1,3 \%$ \\
\hline 7 & 390 & $1,0 \%$ \\
\hline 8 & 242 & $0,6 \%$ \\
\hline 9 & 190 & $0,5 \%$ \\
\hline 10 & 148 & $0,4 \%$ \\
\hline 11 & 115 & $0,3 \%$ \\
\hline 12 & 90 & $0,2 \%$ \\
\hline 13 & 78 & $0,2 \%$ \\
\hline 14 & 78 & $0,2 \%$ \\
\hline 15 & 62 & $0,2 \%$ \\
\hline 16 & 54 & $0,1 \%$ \\
\hline 17 & 45 & $0,1 \%$ \\
\hline 18 & 34 & $0,1 \%$ \\
\hline 19 & 32 & $0,1 \%$ \\
\hline 20 & 32 & $0,1 \%$ \\
\hline 21 & 20 & $0,1 \%$ \\
\hline 22 & 30 & $0,1 \%$ \\
\hline 23 & 14 & $0,0 \%$ \\
\hline 24 & 23 & $0,1 \%$ \\
\hline 25 & 17 & $0,0 \%$ \\
\hline 26 & 10 & $0,0 \%$ \\
\hline 27 & 14 & $0,0 \%$ \\
\hline 28 & 8 & $0,0 \%$ \\
\hline 29 & 14 & $0,0 \%$ \\
\hline 30 & 12 & $0,0 \%$ \\
\hline$>\mathbf{3 0}$ & 182 & $0,5 \%$ \\
\hline Total & 38.237 & $100,0 \%$ \\
\hline
\end{tabular}


Becoña Iglesias ha sido el máximo productor de artículos en revistas españolas $(n=122)$, seguido de dos autores con 63 artículos cada uno: Gual Solé y Villabí Hereter. El porcentaje de artículos en revistas españolas ha sido mayor en Villabí Hereter $(71,6 \%)$, seguido de Becoña Iglesias $(63,5 \%)$ y de Jiménez Ruiz (54,6\%). Los autores que más artículos han publicado en revistas extranjeras han sido Fernández Aranda $(n=183)$, de la Torre $(n=153)$ y Farré Albaladejo $(n=124)$ y Jiménez Murcia $(n=124)$. El porcentaje de artículos en revistas extranjeras fue mayor en Granero Pérez $(97,3 \%)$, Manchón $(95,7 \%)$ y Fernández Aranda $(94,8 \%)$. El ranking de autores por factor de impacto acumulado está liderado por Farré Albaladejo $(F I C=1.019,39)$, seguido de Fernández Aranda $(\mathrm{FIC}=834,67)$ y de la Torre $(\mathrm{FIC}=820,67)$.

En relación con el número de citas recibidas, el ranking en WoS en adicciones está encabezado por Maldonado (Departament de Ciències Experimentals i de la Salut de la Universidad Pompeu Fabra (Barcelona) $(n=4.144)$, seguido de Farré Albaladejo (Catedrático de Farmacología en la Facultad de Medicina de la Universitat Autònoma de Barcelona) $(n=4.020)$ y por Verdejo García $(n=3.677)$. En trastornos de la alimentación destacan Fernández Aranda (2.541), Jiménez Murcia (1.234) y Granero Pérez (1.034). En Scopus se producen algunas variaciones en el ranking anterior pues aquí está liderado en adicciones por Farré Albaladejo $(n=4.283)$, seguido de Verdejo García (Monash Institute of Cognitive and Clinical Neuroscience, Monash University, Melbourne, Australia) $(n=4.212)$ y por de la Torre $(n=3.754)$. En trastornos de la alimentación destacan de nuevo Fernández Aranda (2.808), Jiménez Murcia (1.373) y Granero Pérez (1.158).

\subsection{Productividad e impacto de las instituciones}

De los 16.442 trabajos recuperados, 14.667 (89\%) contenían información sobre las instituciones que habían firmado los trabajos. En la tabla IV se presenta la productividad de las 29 instituciones con más de 200 documentos publicados. De ellas, 13 son universidades, 8 institutos o centros de investigación, 7 hospitales y los restantes de la Agència de Salut Pública de Barcelona. Siete instituciones han publicado más de 500 trabajos. Las instituciones que más artículos han publicado son la Universitat de Barcelona $(n=1.029)$, seguida de la Universitat Autónoma de Barcelona $(n=971)$, la Universitat de València $(n=751)$, la Universidad Politécnica de Madrid $(n=676)$ y Institut Hospital del Mar d'Investigacions Mèdiques (IMIM) $(n=633)$, Universidade de Santiago de Compostela $(n=579)$ y el Instituto de Salud Carlos III $(n=541)$. Estas instituciones lideran la producción en el mismo or- den en las dos áreas analizadas.

Se han desglosado los trabajos según se hayan publicado en revistas españolas o extranjeras. El mayor número de artículos publicados en revistas españolas corresponde a la Universitat Autónoma de Barcelona $(n=151)$, Universitat de Barcelona $(n=132)$ y Universidad Complutense de Madrid $(n=132)$. Sin embargo, los porcentajes de trabajos en estas revistas son mayores para Agència de Salut Pública de Barcelona (ASPB) (35,9\%), Universidad de Oviedo $(28,7 \%)$ y Universidad Miguel Hernández (Elx, Alacant) (25,2\%). En relación con el número de trabajos publicados en revistas extranjeras, el primer puesto lo ocupa la Universitat de Barcelona $(n=754)$, seguida la Universitat Autònoma de Barcelona $(n=718)$ y Institut Hospital del Mar d'Investigacions Mèdiques (IMIM) $(n=538)$. En términos relativos, el primer lugar lo ocupa el Centro de Investigación Biomédica en Red de la Fisiopatología de la Obesidad y Nutrición (CIBEROBN), ya que ha publicado el $88,8 \%$ de los trabajos en revistas extranjeras, seguida del Institut d'Investigació Biomèdica de Bellvitge (IDIBELL) $(87,6 \%)$ y la Universitat Pompeu Fabra (87,3\%). Debe tenerse en cuanta que en 1.610 trabajos $(9.8 \%)$ no ha sido posible identificar el país de procedencia de la revista por tratarse, habitualmente, de revistas desaparecidas.

Las instituciones que han recibido más citas en WoS en el área de las adicciones han sido la Universitat de Barcelona $(n=15.554)$, seguida de Institut Hospital del Mar d'Investigacions Mèdiques (IMIM) $(n=15.057)$ y de la Universitat Autónoma de Barcelona $(n=13.532)$. En el área de los trastornos de la alimentación destacan también Universitat de Barcelona $(n=4.132)$, Universitat Autónoma de BarceIona $(n=3.114)$ y Hospital Universitari de Bellvitge (3.022). En Scopus las instituciones que acapararon un mayor número de citas en adicciones fueron Universitat de Barcelona $(n=16.977)$, seguida del Institut Hospital del Mar d'Investigacions Mèdiques (IMIM) $(n=16.010)$ y de la Universitat Autónoma de Barcelona $(n=15.607)$. Por último, en trastornos de la alimentación, las instituciones más citadas han sido Universitat Autónoma de Barcelona $(n=3.587)$, Hospital Universitari de Bellvitge (3.358) y Universitat de Barcelona (3.046).

\section{TRABAJOS MÁS CITADOS}

Los 18 artículos que han recibido más de 350 citas en WoS se presentan en la tabla V. El primer trabajo se acerca a las mil citas (946) y fue publicado en 2008 en la revista Nature por Thorgeirsson (deCODE Genetics, Reikiavik, Islandia) y otros, en el que participaron investigadores españoles como 


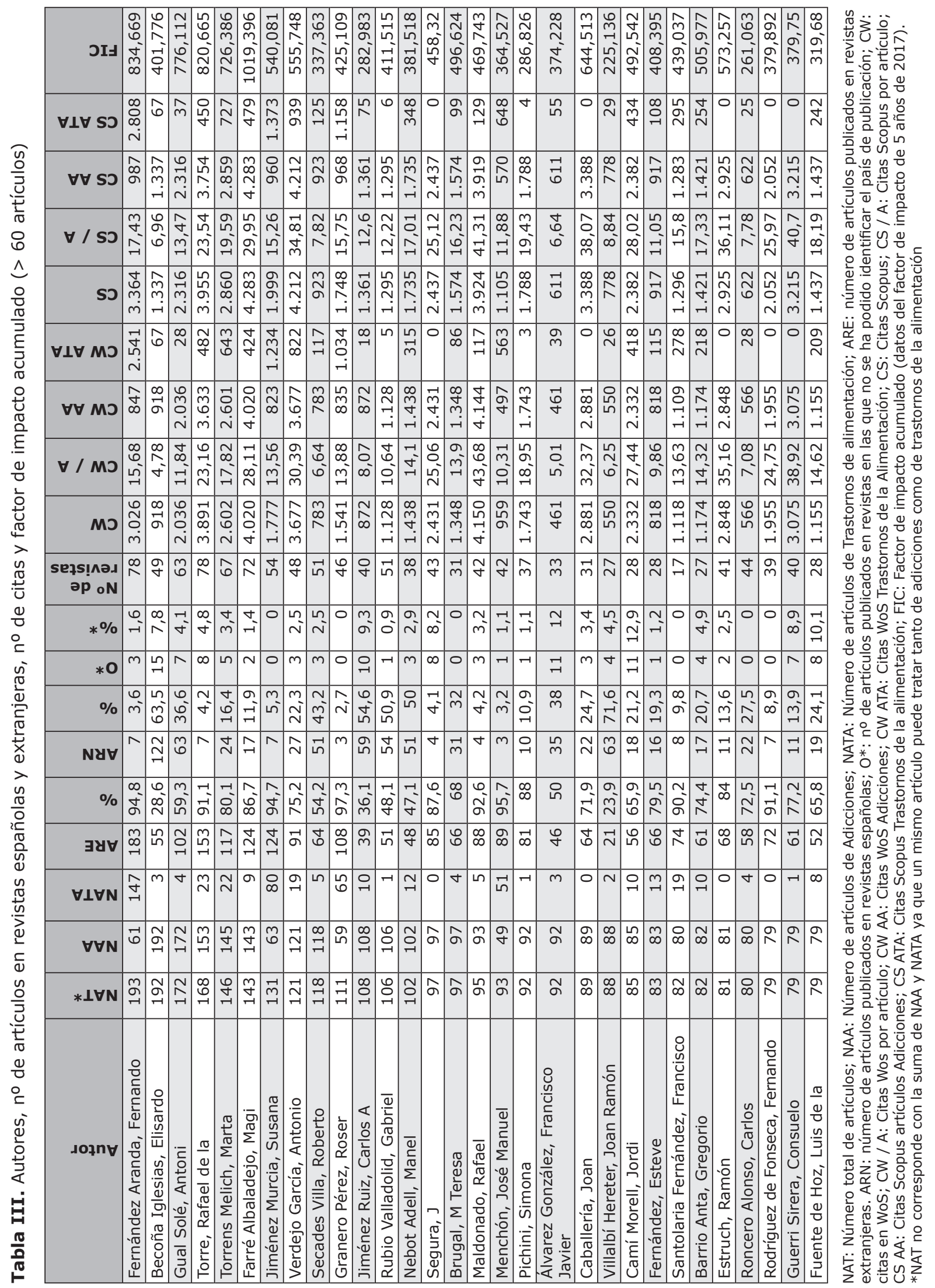




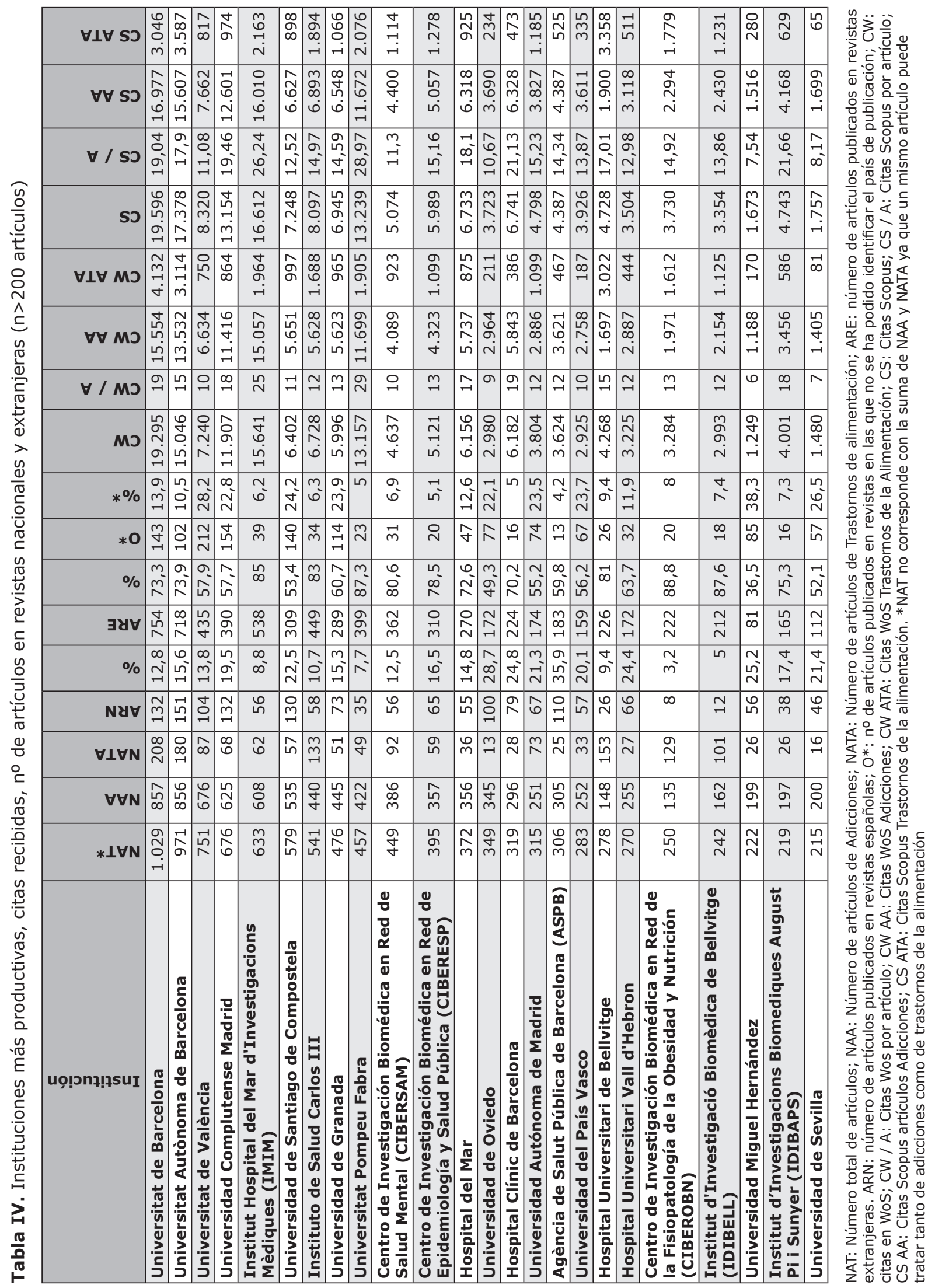


Isla y Vidal (Departamento de Oncología Médica del Hospital Universitario de Zaragoza), Asín (Instituto de Nanotecnología de Aragón, Zaragoza), Sáez (Instituto de la Salud, Zaragoza) y Murillo (Hospital Reina Sofía, División de Oncología Médica, Tudela, Zaragoza). Este trabajo también obtuvo un mayor índice de citas por año. El segundo trabajo más citado se publicó en 2003 por Green y otros (742 citas) en Pharmacological Reviews y en él participó Colado, del Departamento de Farmacología de la Facultad de Medicina de la Universidad Complutense de Madrid. Merece destacarse aquí que el tercer trabajo tiene como primer firmante a un autor español: Verdejo-García (Unidad de Investigación e Farmacología, Institut Municipal d'Investigació Mèdica, Barcelona), publicado en 2008 en Neuroscience and Biobehavioral Reviews. Las citas por año producen algunas pequeñas variaciones en el ranking, pero sigue encabezado por el trabajo de Thorgeirsson y otros, seguido por el de Gao y Bataller (Unidad Hepática, Institut de Malalties Digestives i Metabòliques, Hospital Clinic, Institut d'Investigació Biomèdiques August Pi i Sunyer (IDIBAPS), Barcelona), publicado en 2011 en Gastroenterology, y por el de Evans y otros. Trece de los 18 trabajos más citados son artículos originales y cinco son revisiones. Las temáticas tratadas en el conjunto son el tabaquismo (en 7 trabajos), el alcoholismo (7), los trastornos de la alimentación (2), los trastornos adictivos en general (2) y, por último, las drogas de diseño, el juego patológico y la cocaína con un trabajo, respectivamente. Dos trabajos trataban conjuntamente sobre alcohol y tabaco, y uno sobre Trastorno adictivo y Trastornos de la alimentación.

\section{INDICADORES DE COLABORACIÓN CIEN- TÍFICA}

\subsection{Colaboración entre autores}

El $85,8 \%$ de los trabajos han sido realizados en colaboración, es decir, fueron firmados por dos o más autores. La mayor parte de los trabajos han sido firmados por un autor $(n=2.318,14,2 \%)$, pero también predominan los firmados por dos $(n=1.971 ; 12,1 \%)$, tres $(n=2368 ; 14,5 \%)$ o cuatro autores $(n=2.436 ; 14,9 \%)$. El índice de colaboración entre autores (índice firmas/trabajo) a lo largo del periodo ha aumentado progresivamente, pasando de 1,56 autores por trabajo en el periodo 1962-1972 a 7,08 (Figura 3) autores por trabajo en el quinquenio 2013-2017. La media o índice global del periodo en las dos áreas ha sido de $5,19$ autores por trabajo ( $D S=5,89)$. Este índice se ha calculado excluyendo los cuatro trabajos que sobrepasaban los 200 firmantes. El índice es ligeramente mayor en el área de trastornos de la ali- mentación que en el de las adicciones (5,70 frente a 5,16$)$.

Figura 3. Evolución del índice de firmantes por trabajo por periodos

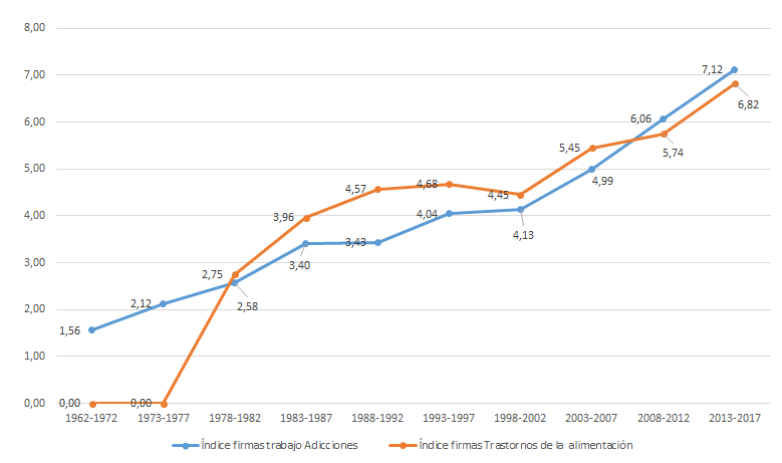

En las redes de colaboración entre autores han participado 470 investigadores, de los que 115 $(24,5 \%)$ son extranjeros. La red que tiene un mayor número de integrantes (90 investigadores, figura 4) tiene como autor central a Fernández Aranda (Unidad de Trastornos de la Alimentación del Servicio de Psiquiatría del Hospital Universitario de Bellvitge, Hospitalet de Llobregat, Barcelona). Este investigador tiene fuertes conexiones de colaboración con tres investigadores: Granero Pérez (Departamento de Psicobiología y Metodología, Universitat Autònoma de Barcelona), Jiménez Murcia (Unidad de Juego Patológico del Hospital Universitario de Bellvitge) y Menchón (CIBERSAM, Hospital Universitario de Bellvitge / Fundación IDIBELL). En esta red se distinguen hasta tres subgrupos (en la parte inferior de la red): el de De la Torre, Camí Morell y Farré Albaladejo (en el centro de la figura, integrados mayoritariamente en el Instituto Hospital de Mar de Investigaciones Médicas-IMIM); el de Barrio Anta y de De la Fuente de Hoz (Instituto de Salud Carlos III, Madrid); y el de Esteve Fernández (Unidad de Control del Tabaquismo, Instituto Catalán de Oncología y Universitat de Barcelona).

En la figura 5 se presentan los coautores de la red de 80 colaboradores. Se puede apreciar que la mayor parte de ellos son autores extranjeros, y que los españoles que han colaborado con ellos son Antoni Agudo Trigueros (Unidad de Nutrición y Cáncer. Institut d'Investigació Biomèdica de BeIlvitge, Barcelona), Barricarte Gurrea y Ardanaz Aicua (Servicio de Epidemiología y Prevención Sanitaria de Navarra) y Quirós Navarro, entre otros. En esta red, el investigador que más se relaciona con otros es Riboli (Director of the School of Public Health, Imperial College London). Esta red 
Tabla V. Trabajos más citados en Web of Science (con más de 350 citas)

\begin{tabular}{|c|c|c|c|c|c|c|c|}
\hline y & $\frac{0}{3}$ & 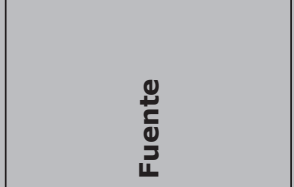 & 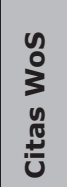 & 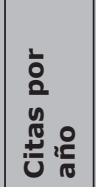 & 은 & 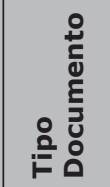 & $\underbrace{\frac{\pi}{2}}_{\substack{\pi \\
\frac{\pi}{2}}}$ \\
\hline $\begin{array}{l}\text { Thorgeirsson, TE; } \\
\text { Geller, F; Sulem, P; } \\
\text { Rafnar, T; Wiste, A; } \\
\text { Magnusson, KP; y } \\
\text { otros } \\
\end{array}$ & $\begin{array}{l}\text { A variant associated with nicotine } \\
\text { dependence, lung cancer and } \\
\text { peripheral arterial disease }\end{array}$ & $\begin{array}{l}\text { Nature 2008; } \\
\text { 452(7187): 638-U9 }\end{array}$ & 946 & 94,6 & 2008 & Article & Tabaco \\
\hline $\begin{array}{l}\text { Green, AR; } \\
\text { Mechan, AO; } \\
\text { Elliott, JM; } \\
\text { O'Shea, E; } \\
\text { Colado, MI }\end{array}$ & $\begin{array}{l}\text { The pharmacology and } \\
\text { clinical pharmacology of } \\
3,4-\text { methylenedioxymethamphetamine } \\
\text { (MDMA, "ecstasy") }\end{array}$ & $\begin{array}{l}\text { Pharmacological } \\
\text { Reviews 2003; } \\
\text { 55(3): 463-508 }\end{array}$ & 742 & 49,47 & 2003 & Review & $\begin{array}{l}\text { Drogas de } \\
\text { diseño }\end{array}$ \\
\hline $\begin{array}{l}\text { Verdejo-Garcia, } \\
\text { A; Lawrence, A]; } \\
\text { Clark, L }\end{array}$ & $\begin{array}{l}\text { Impulsivity as a vulnerability marker } \\
\text { for substance-use disorders: Review } \\
\text { of findings from high-risk research, } \\
\text { problem gamblers and genetic } \\
\text { association studies }\end{array}$ & $\begin{array}{l}\text { Neuroscience and } \\
\text { Biobehavioral } \\
\text { Reviews 2008; } \\
\text { 32(4): } 777-810\end{array}$ & 595 & 59,5 & 2008 & Review & $\begin{array}{l}\text { Juego } \\
\text { patológico }\end{array}$ \\
\hline $\begin{array}{l}\text { Muscaritoli, } \\
\text { M; Anker, SD; } \\
\text { Argiles, J; Aversa, } \\
\text { Z; Bauer, JM; } \\
\text { Biolo, G; y otros }\end{array}$ & $\begin{array}{l}\text { Consensus definition of sarcopenia, } \\
\text { cachexia and pre-cachexia: Joint } \\
\text { document elaborated by Special } \\
\text { Interest Groups (SIG) "cachexia- } \\
\text { anorexia in chronic wasting diseases" } \\
\text { and "nutrition in geriatrics" }\end{array}$ & $\begin{array}{l}\text { Clinical Nutrition } \\
2010 ; 29(2): 154- \\
159\end{array}$ & 559 & 69,88 & 2011 & Review & $\begin{array}{l}\text { Trastornos } \\
\text { de la } \\
\text { alimentación }\end{array}$ \\
\hline Gao, B; Bataller, R & $\begin{array}{l}\text { Alcoholic Liver Disease: Pathogenesis } \\
\text { and New Therapeutic Targets }\end{array}$ & $\begin{array}{l}\text { Gastroenterology } \\
2011 ; 141(5): 1572- \\
1585\end{array}$ & 559 & 79,86 & 2010 & Article & Alcohol \\
\hline $\begin{array}{l}\text { Beral, V; } \\
\text { Hamajima, N; } \\
\text { Hirose, K; Rohan, } \\
\text { T; Calle, EE; } \\
\text { Heath, CW; y otros }\end{array}$ & $\begin{array}{l}\text { Alcohol, tobacco and breast cancer - } \\
\text { collaborative reanalysis of individual } \\
\text { data from } 53 \text { epidemiological studies, } \\
\text { including } 58515 \text { women with breast } \\
\text { cancer and } 95067 \text { women without the } \\
\text { disease }\end{array}$ & $\begin{array}{l}\text { British Journal } \\
\text { of Cancer 2002; } \\
\text { 87(11): 1234-1245 }\end{array}$ & 552 & 34,5 & 2002 & Article & $\begin{array}{l}\text { Alcohol y } \\
\text { tabaco }\end{array}$ \\
\hline $\begin{array}{l}\text { Salamone, JD; } \\
\text { Correa, M; Farrar, } \\
\text { A; Mingote, SM } \\
\end{array}$ & $\begin{array}{l}\text { Effort-related functions of nucleus } \\
\text { accumbens dopamine and associated } \\
\text { forebrain circuits }\end{array}$ & $\begin{array}{l}\text { Psychopharmacology } \\
2007 ; 191(3): 461- \\
482\end{array}$ & 526 & 47,82 & 2007 & Review & $\begin{array}{l}\text { Trastorno } \\
\text { adictivo }\end{array}$ \\
\hline $\begin{array}{l}\text { Teo, KK; Ounpuu, } \\
\text { S; Hawken, S; } \\
\text { Pandey, MR; } \\
\text { Valentin, V; y } \\
\text { otros } \\
\end{array}$ & $\begin{array}{l}\text { Tobacco use and risk of myocardial } \\
\text { infarction in } 52 \text { countries in the } \\
\text { INTERHEART study: a case-control } \\
\text { study }\end{array}$ & $\begin{array}{l}\text { Lancet 2006; } \\
\text { 368(9536): 647-658 }\end{array}$ & 507 & 42,25 & 2006 & Article & Tabaco \\
\hline $\begin{array}{l}\text { Everitt, B]; } \\
\text { Parkinson, } \\
\text { JA; Olmstead, } \\
\text { MC; Arroyo, } \\
\text { M; Robledo, P; } \\
\text { Robbins, TW } \\
\end{array}$ & $\begin{array}{l}\text { Associative processes in addiction and } \\
\text { reward - The role of amygdala-ventral } \\
\text { striatal subsystems }\end{array}$ & $\begin{array}{l}\text { Annals of the New } \\
\text { York Academy of } \\
\text { Sciences } 1999 ; \\
877(): 412-438\end{array}$ & 500 & 26,32 & 1999 & Article & $\begin{array}{l}\text { Trastorno } \\
\text { adictivo y } \\
\text { Trastornos } \\
\text { de la } \\
\text { alimentación }\end{array}$ \\
\hline $\begin{array}{l}\text { Urbanomarquez, } \\
\text { A; Estruch, R; } \\
\text { Navarrolopez, F; } \\
\text { Grau, Jm; Mont, L; } \\
\text { Rubin, E } \\
\end{array}$ & $\begin{array}{l}\text { The effects of alcoholism on skeletal } \\
\text { and cardiac-muscle }\end{array}$ & $\begin{array}{l}\text { New England Journal } \\
\text { of Medicine 1989; } \\
320(7): 409-415\end{array}$ & 483 & 16,66 & 1989 & Article & Alcohol \\
\hline $\begin{array}{l}\text { Hashibe, M; } \\
\text { Brennan, P; } \\
\text { Benhamou, S; } \\
\text { Castellsague, } \mathrm{X} \text {; } \\
\text { Chu, C; Curado, } \\
\text { MP; y otros } \\
\end{array}$ & $\begin{array}{l}\text { Alcohol drinking in never users of } \\
\text { tobacco, cigarette smoking in never } \\
\text { drinkers, and the risk of head and } \\
\text { neck cancer: Pooled analysis in the } \\
\text { international head and neck cancer } \\
\text { epidemiology consortium }\end{array}$ & $\begin{array}{l}\text { Jnci-journal of the } \\
\text { National Cancer } \\
\text { Institute 2007; } \\
99(10): 777-789\end{array}$ & 451 & 41 & 2007 & Article & Alcohol \\
\hline $\begin{array}{l}\text { Furberg, H; Kim, } \\
\text { Y; Dackor, J; } \\
\text { Boerwinkle, E; } \\
\text { Franceschini, N; } \\
\text { Ardissino, D; y } \\
\text { otros }\end{array}$ & $\begin{array}{l}\text { Genome-wide meta-analyses identify } \\
\text { multiple loci associated with smoking } \\
\text { behavior }\end{array}$ & $\begin{array}{l}\text { Nature Genetics } \\
2010 ; 42(5): 441- \\
\text { U134 }\end{array}$ & 448 & 56 & 2010 & Article & Tabaco \\
\hline
\end{tabular}




\begin{tabular}{|c|c|c|c|c|c|c|c|}
\hline $\begin{array}{l}y \\
\frac{1}{0} \\
\frac{3}{3} \\
\frac{2}{4}\end{array}$ & $\frac{0}{3}$ & 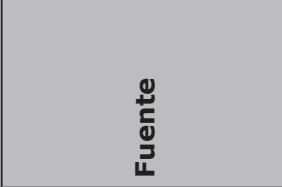 & 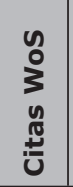 & 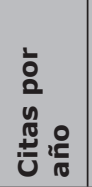 & ㅇํㄴ & 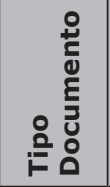 & $\frac{\pi}{2}$ \\
\hline $\begin{array}{l}\text { Hernan, MA; } \\
\text { Takkouche, B; } \\
\text { Caamano-Isorna, } \\
\text { F; Gestal-Otero, J] }\end{array}$ & $\begin{array}{l}\text { A meta-analysis of coffee drinking, } \\
\text { cigarette smoking, and the risk of } \\
\text { Parkinson's disease }\end{array}$ & $\begin{array}{l}\text { Annals of Neurology } \\
2002 ; 52(3): 276- \\
284\end{array}$ & 408 & 25,5 & 2002 & Article & $\begin{array}{l}\text { Alcohol y } \\
\text { tabaco }\end{array}$ \\
\hline $\begin{array}{l}\text { Thorgeirsson, TE; } \\
\text { Gudbjartsson, DF; } \\
\text { Surakka, I; Vink, } \\
\text { JM; Amin, N; } \\
\text { Geller, F; y otros } \\
\end{array}$ & $\begin{array}{l}\text { Sequence variants at CHRNB3- } \\
\text { CHRNA6 and CYP2A6 affect smoking } \\
\text { behavior }\end{array}$ & $\begin{array}{l}\text { Nature Genetics } \\
2010 ; 42(5): 448- \\
\text { U135 }\end{array}$ & 400 & 50 & 2010 & Article & Tabaco \\
\hline $\begin{array}{l}\text { Eckardt, MJ; File, } \\
\text { SE; Gessa, GL; } \\
\text { Grant, KA; Guerri, } \\
\text { C; Hoffman, PL; y } \\
\text { otros }\end{array}$ & $\begin{array}{l}\text { Effects of moderate alcohol } \\
\text { consumption on the central nervous } \\
\text { system }\end{array}$ & $\begin{array}{l}\text { Alcoholism-clinical } \\
\text { and Experimental } \\
\text { Research 1998; } \\
\text { 22(5): } 998-1040\end{array}$ & 399 & 19,95 & 1998 & Review & Alcohol \\
\hline $\begin{array}{l}\text { Cavelaars, AEJM; } \\
\text { Kunst, AE; Geurts, } \\
\text { JJM; Crialesi, } \\
\text { R; Grotvedt, L; } \\
\text { Helmert, U; y } \\
\text { otros } \\
\end{array}$ & $\begin{array}{l}\text { Educational differences in smoking: } \\
\text { international comparison }\end{array}$ & $\begin{array}{l}\text { British Medical } \\
\text { Journal 2000; } \\
320(7242): 1102- \\
1107\end{array}$ & 379 & 21,06 & 2000 & Article & Tabaco \\
\hline $\begin{array}{l}\text { Valjent, E; Corvol, } \\
\text { JC; Pages, C; } \\
\text { Besson, MJ; } \\
\text { Maldonado, R; } \\
\text { Caboche, J }\end{array}$ & $\begin{array}{l}\text { Involvement of the extracellular } \\
\text { signal-regulated kinase cascade for } \\
\text { cocaine-rewarding properties }\end{array}$ & $\begin{array}{l}\text { Journal of } \\
\text { Neuroscience 2000; } \\
\text { 20(23): 8701-8709 }\end{array}$ & 373 & 20,72 & 2000 & Article & Cocaína \\
\hline $\begin{array}{l}\text { Degenhardt, } \\
\text { L; Chiu, WT; } \\
\text { Sampson, N; } \\
\text { Kessler, RC; } \\
\text { Anthony, JC; } \\
\text { Angermeyer, M; y } \\
\text { otros }\end{array}$ & $\begin{array}{l}\text { Toward a global view of alcohol, } \\
\text { tobacco, cannabis, and cocaine use: } \\
\text { Findings from the WHO World Mental } \\
\text { Health Surveys }\end{array}$ & $\begin{array}{l}\text { PLOS Medicine } \\
2008 ; 5(7): 1053- \\
1067\end{array}$ & 364 & 36,4 & 2008 & Article & Alcohol \\
\hline
\end{tabular}

Figura 4. Red de colaboración entre autores con un mayor número de integrantes (90 coautores)

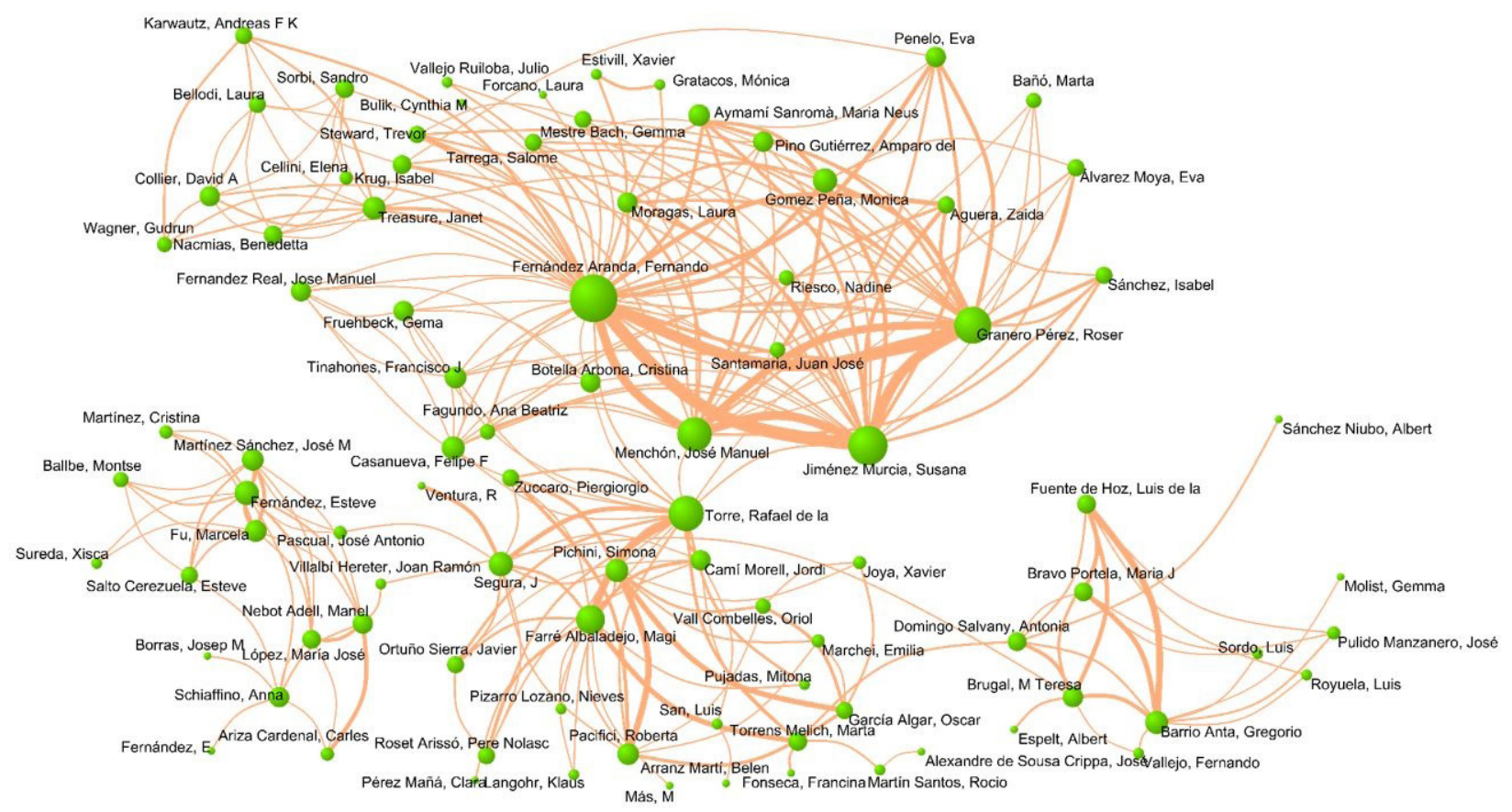


Figura 5. Red de colaboración entre autores (80 coautores)

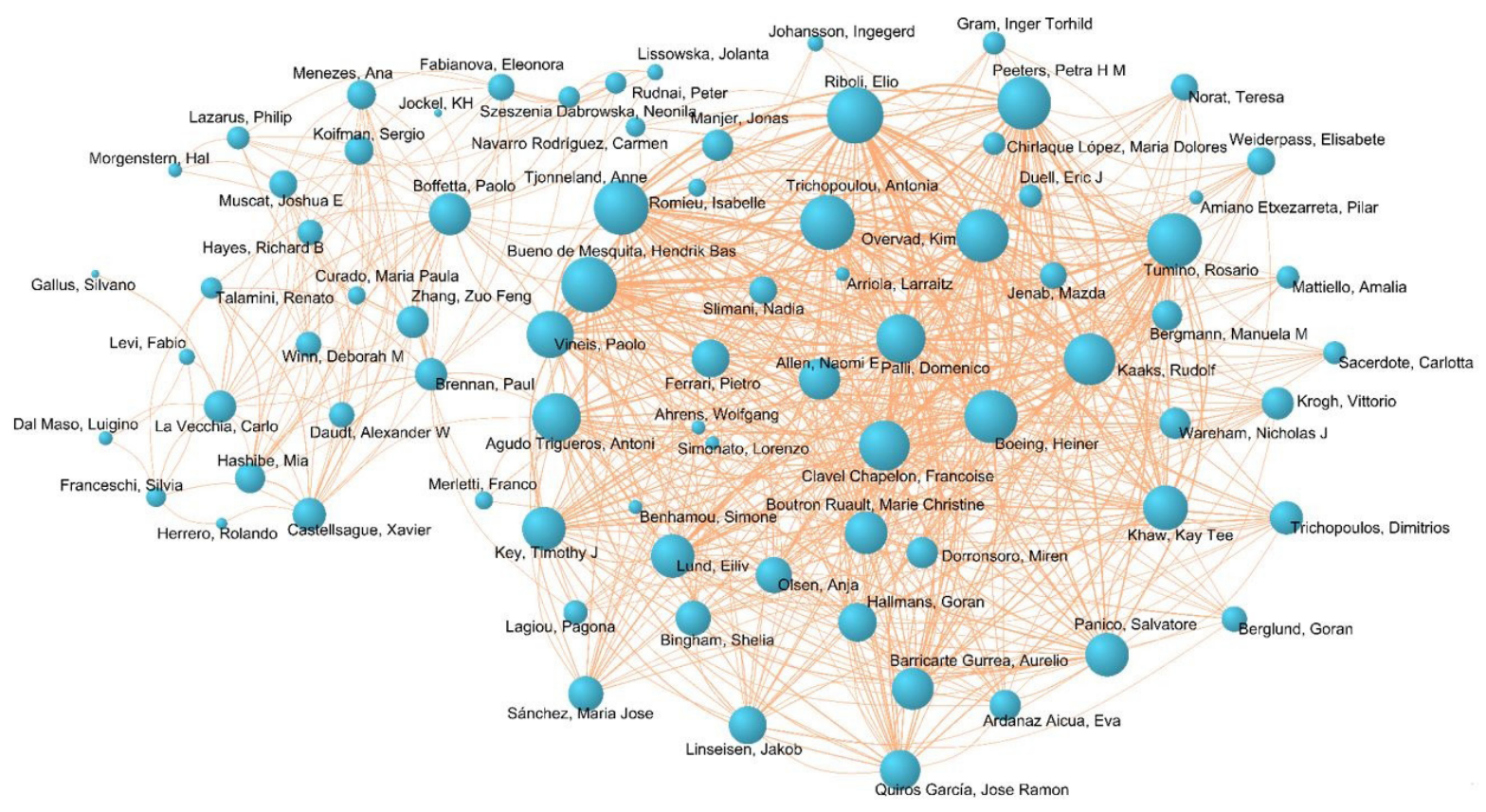

Figura 6. Red de colaboración entre autores (11 y 10 coautores)
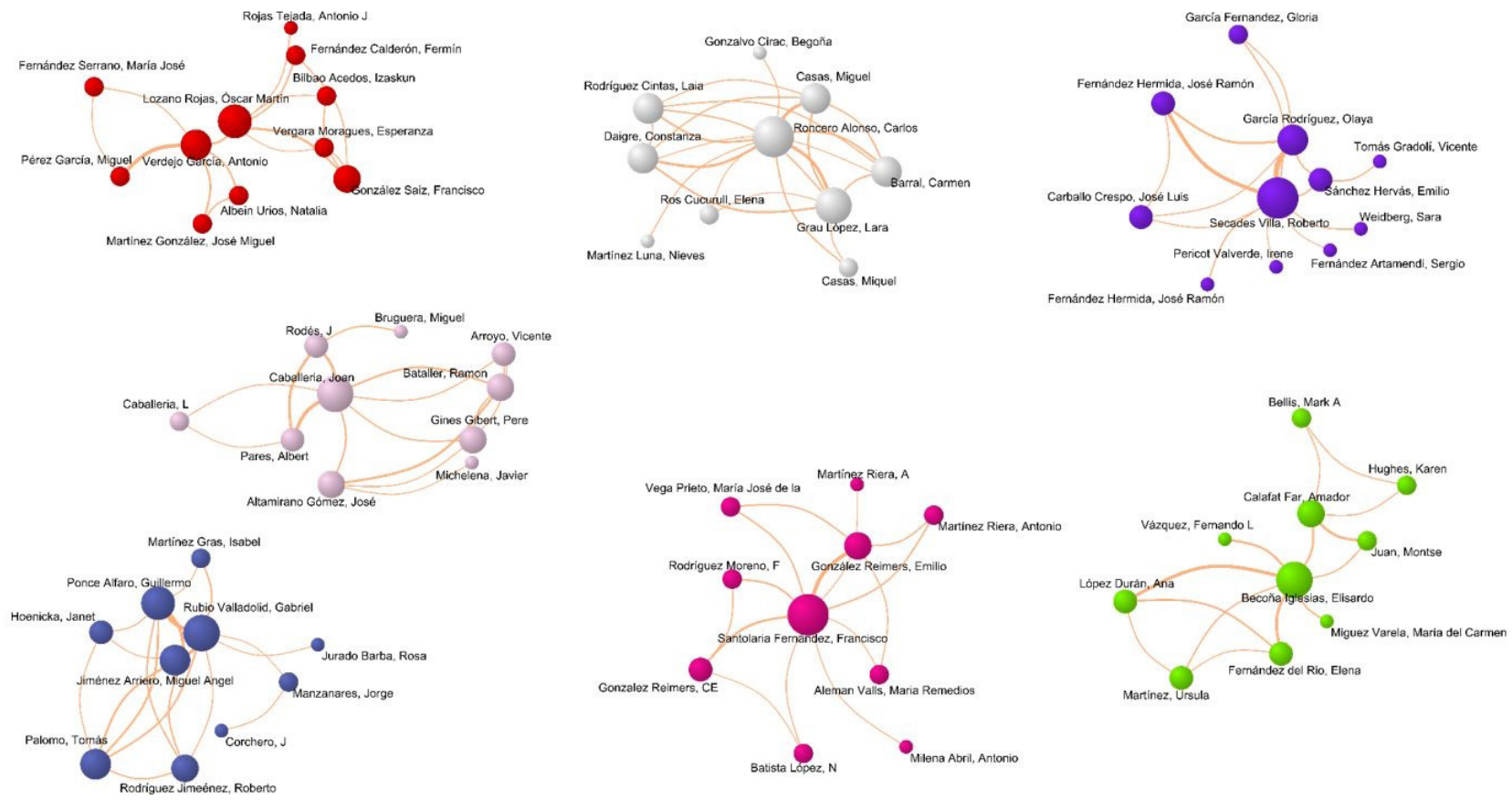

está más cohesionada que la de 90 componentes y tiene un núcleo satélite al que se conecta por medio de Brenan y Boffeta (International Agency for Research on Cancer, Lyon, Francia).

La siguiente figura muestra las dos redes de 11 coautores y las cinco redes de 10 integrantes (figura 6). La primera de ellas incluye dos autores centrales, Martín Lozano Rojas (Departamento de Psicología Clínica, Experimental y Social. Universidad de Huelva) y Verdejo García (Pharmacology Research Unit, 
Institut Municipal d'Investigació Mèdica, Barcelona). Otras redes de la imagen tiene como autores centrales a Roncero Alonso (Servicio de Psiquiatría Complejo Asistencial Universitario de Salamanca); Secades Villa (Departamento de Psicología de la Universidad de Oviedo); Caballeria Rovira (Hospital Clínic de Barcelona); Rubio Valladolid (Departamento de Psiquiatría del Hospital Universitario 12 de octubre); Santolaria Fernández (Departamento de Medicina Interna, Dermatología y Psiquiatría, Hospital Universitario de Canarias), Becoña Iglesias (Departamento de Psicología Clínica y Psicobiología de la Universidade de Santiago de Compostela).

En la figura complementaria 1 https://zenodo. org/record/4746351\#.YJlJ5qHtaUk se muestran 9 redes más pequeñas: una de 8 coautores, dos de siete y seis de seis autores. Entre estas redes, algunas destacan por tener un autor con mayor centralidad o número de conexiones, como la de Gual Solé (barnaclínic+, Hospital Clínic de Barcelona), el de Pozo (Bioanalysis Research Group, Parc de Salut Mar, Barcelona), la de Rodríguez de Fonseca (Instituto de Investigación Biomédica de Málaga-IBIMA) y la de Miñarro López y Aguilar Calpe (Departamento de Psicobiología, Universitat de València), entre otros. Por último, en la figura complementaria 2 https://zenodo.org/record/4746351\#.YJlJ$5 q H$ taUk se dibujan 15 redes adicionales, nueve de 5 autores y seis de 4 autores. Algunos autores que destacan en estas redes por su centralidad son: Laso Guzmán (Servicio de Medicina Interna, Complejo Asistencial Universitario de Salamanca); Valderrama Zurián (Universitat de València) y Cervera Martínez (Hospital Clínic de València); Flores (UNED-Universidad Nacional de Educación a Distancia); Maldonado (Departament de Ciències Experimentals i de la Salut de la Universidad Pompeu Fabra, Barcelona); González Quintela (Complejo Hospitalario Universitario de Santiago de Compostela, Universidad de Santiago de Compostela); Rodríguez Artalejo (Universidad Autónoma de Madrid); Pérez de los Cobos Peris (Hospital de la Santa Creu i Sant Pau, Barcelona).

En lo que respecta a la topología de las redes, la que tiene un mayor número de integrantes (90) es una red descentralizada y sociocéntrica en forma de malla en la que la mayoría de los nodos se conectan entre sí. En ella se aprecia la existencia de nodos vinculadores (como el que corresponde a Rafael de la Torre y José Manuel Menchón), que actúan de puentes y propician tanto la distribución de información como el tamaño de la red. La red compuesta por 80 autores, aunque también es una red sociocéntrica, es más distributiva y casi conecta a todos sus integrantes; en ella no destaca significativamente un nodo de los demás, por lo que en este tipo de red la información puede fluir más rápidamente que en la anterior. Las redes de 11 y 10 integrantes suelen tener una topología centralizada y en las de menos integrantes (10 o menos), las representaciones de la red son más heterogéneas.

\subsection{Colaboración entre instituciones}

La figura 7 presenta los datos sobre la colaboración institucional dividida en dos modalidades, nacional e internacional, así como la inexistencia de colaboración, tanto en toda la población de trabajos (7a), como en las adicciones (7b) y en trastornos de la alimentación (7c). Como puede apreciarse, el porcentaje de artículos en los que se ha producido colaboración entre dos o más instituciones nacionales ha aumentado progresivamente y pasado del $16,7 \%$ en el periodo $1962-1972$ al 73,2\% en el quinquenio 2013-2017. De forma paralela ha aumentado la colaboración con instituciones extranjeras, que ha pasado del $16,7 \%$ en el periodo $1962-1972$ al $35,7 \%$ en el quinquenio 2013-2017. Por último, los trabajos publicados sin colaboración institucional, han pasado del 66,7\% en el periodo inicial al $20,6 \%$ en el periodo final. Como puede apreciarse, el porcentaje de trabajos en los que no existe colaboración institucional ha disminuido progresivamente desde la década de los 60 , situándose en el $20,6 \%$ de los trabajos en el quinquenio 2013-2107. El porcentaje de colaboración internacional en el último periodo es mayor en los trabajos sobre adicciones que sobre los trastornos de la alimentación (20,80\% frente a $15,70 \%)$. En el caso de la colaboración nacional ocurre lo contrario, ya que es mayor en trastornos de la alimentación $(79,90)$ que en adicciones $(72,70)$.

Las instituciones extranjeras más destacadas que han colaborado con otras españolas en las dos áreas (tabla VI) han sido la International Agency for Research on Cancer Lyon (IARC) $(n=158)$, seguida de Kings College of London $(n=142)$ y Karolinska Institutet de Suecia $(n=142)$. En el área de las adicciones, las instituciones más destacas han sido la International Agency for Research on Cancer Lyon (IARC) $(n=157)$, seguida de Karolinska Institutet (137) y de Imperial College London (115). En el área de los trastornos de la alimentación sobresalen Kings College of London $(n=46)$, University of North Carolina Chapel Hill (Estados Unidos; $n=20$ ) y Columbia University (Estados Unidos; $n=13$ ). Como puede apreciarse, se trata de instituciones de países con los que colaboran los centros españoles, como Estados Unidos, Francia, Reino Unido, Italia, Alemania y Países Bajos, entre otros. No se ha identificado ninguna institución de América Latina con 60 o más artículos. Las institu- 
Figura 7a. Evolución de la colaboración institucional por periodos en las áreas de adicciones y de trastornos de la alimentación

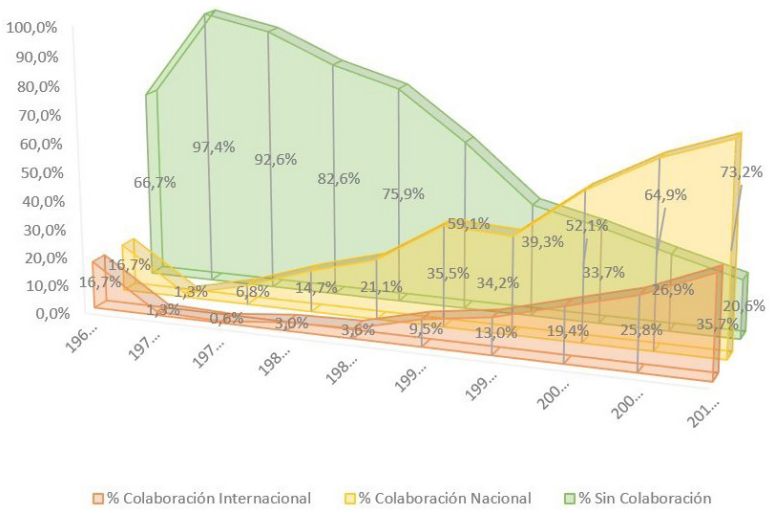

Figura 7b. Evolución de la colaboración institucional por periodos en el área de adicciones

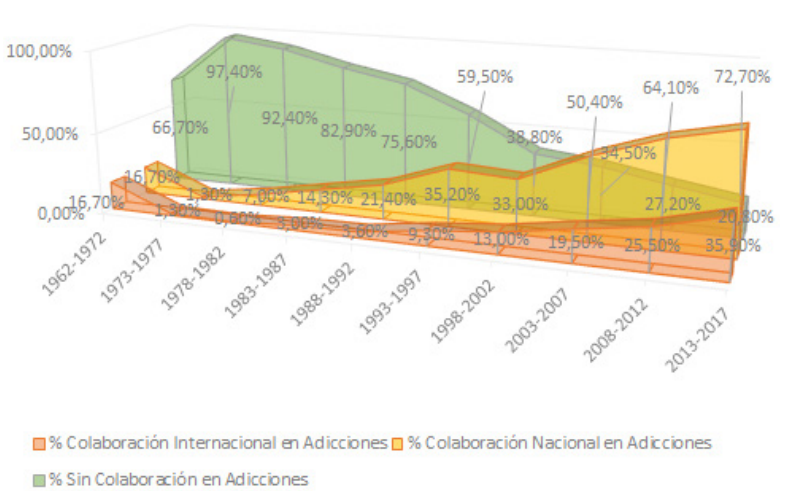

Figura 7c. Evolución de la colaboración institucional por periodos en el área de trastornos de la alimentación

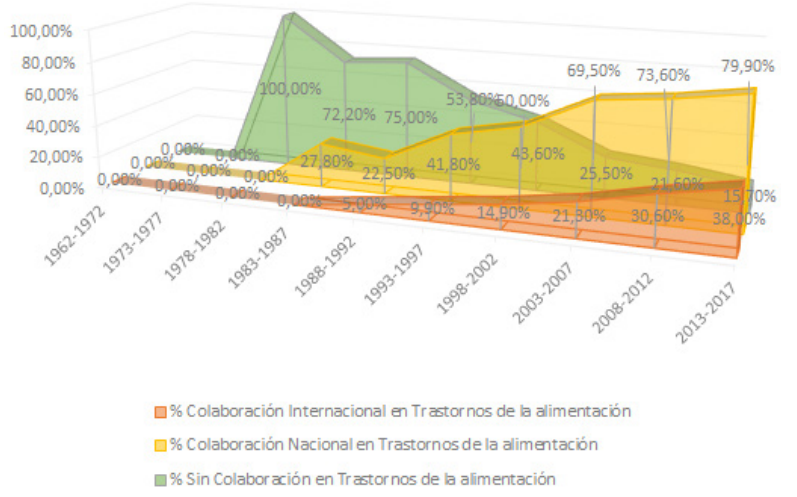

ciones de América Latina con las que más trabajos se ha publicado en colaboración han sido la Universidade de São Paulo $(n=53)$ de Brasil, la Universidad de Buenos Aires $(n=33)$ de Argentina, el Instituto Nacional de Psiquiatría Ramón de la Fuente Muñiz $(n=20)$ de México, la Universidad Nacional de Córdoba $(n=17)$ de Argentina y el Hospital de Clínicas de Porto Alegre (HCPA) $(n=15)$ de Brasil.

En relación con la red de colaboración entre instituciones, con el umbral establecido se forma una compleja red integrada por 53 instituciones nacionales y extranjeras en la que se pueden apreciar varios subgrupos y otros que actúan como nexos de unión entre las redes (figura 8). Por ejemplo, CIBERESP, Institut Català d'Oncologia y IDIBELL enlazan las instituciones de la parte superior de la red con las extranjeras de la parte inferior (entre ellas, International Agency for Research on Cancer-IARC, Imperial College of London, University of
Oxford y University Medical Center of Utrecht) y con algunas españolas como la Escuela de Salúd Pública-EASP. El Instituto de Salud Carlos III sirve de puente entre las instituciones catalanas y las valencianas (Universitat de València, Universitat Jaume I, Hospital Clínic Universitari de València y Hospital Universitari i Politècnic La Fe), y también con las de Madrid (Universidad Complutense de Madrid, Universidad Autónoma de Madrid y Hospital Universitario 12 de Octubre).

Las principales colaboraciones en esta red son: Institut Hospital del Mar d'Investigacions Mèdiques (IMIM) con Universitat Pompeu Fabra (273 colaboraciones), con Universitat Autònoma de BarceIona (243 colaboraciones) y con Hospital del Mar (211 colaboraciones); Universitat de Barcelona con Institut d'Investigació Biomèdica de Bellvitge (IDIBELL) (211) y con Institut d'Investigacions Biomediques August Pi i Sunyer (IDIBAPS) (209); 
Tabla VI. Instituciones extranjeras que han colaborado con las españolas (60 o más colaboraciones)

\begin{tabular}{|c|c|c|c|c|}
\hline Institución & País & NAT* & NAA & NATA \\
\hline International Agency for Research on Cancer Lyon (IARC) & Francia & 158 & 157 & 1 \\
\hline Kings College London & Reino Unido & 142 & 100 & 46 \\
\hline Karolinska Institutet & Suecia & 142 & 137 & 10 \\
\hline Imperial College London & Reino Unido & 118 & 115 & 5 \\
\hline $\begin{array}{l}\text { Institut National de la Santé et de la Recherche Médicale } \\
\text { (INSERM) }\end{array}$ & Francia & 118 & 112 & 6 \\
\hline Istituto Superiore di Sanità & Italia & 110 & 110 & 2 \\
\hline University of Athens & Grecia & 108 & 107 & 3 \\
\hline German Cancer Research Center (DKFZ) & Alemania & 106 & 106 & 1 \\
\hline Harvard University & Estados Unidos & 93 & 87 & 9 \\
\hline Columbia Universiy & Estados Unidos & 87 & 86 & 13 \\
\hline University of Cambridge & Reino Unido & 86 & 85 & 3 \\
\hline University Medical Center Utrecht & Países Bajos & 85 & 82 & 3 \\
\hline University of Oxford & Reino Unido & 83 & 82 & 1 \\
\hline $\begin{array}{l}\text { Netherlands National Institute for Public Health and the } \\
\text { Environment (RIVM) }\end{array}$ & Países Bajos & 81 & 81 & 0 \\
\hline University of Turin & Italia & 81 & 80 & 2 \\
\hline University of Southern California & Estados Unidos & 77 & 75 & 3 \\
\hline University of North Carolina Chapel Hill & Estados Unidos & 76 & 61 & 20 \\
\hline Danish Cancer Society Research Center & Dinamarca & 75 & 75 & 0 \\
\hline University of Milan & Italia & 75 & 72 & 6 \\
\hline Institute Gustave Roussy & Francia & 69 & 69 & 0 \\
\hline Julius Center for Health Sciences and Primary Care & Países Bajos & 65 & 65 & 0 \\
\hline German Institute of Human Nutrition Potsdam-Rehbruecke & Alemania & 65 & 65 & 0 \\
\hline IRCCS Ist Recerche Farmacol Mario Negri & Italia & 63 & 63 & 4 \\
\hline University of Tromso Arctic University of Norway & Noruega & 62 & 60 & 2 \\
\hline Lund University & Suecia & 62 & 57 & 6 \\
\hline Mount Sinai School of Medicine New York & Estados Unidos & 61 & 55 & 9 \\
\hline
\end{tabular}

NAT: Número total de artículos; NAA: Número de artículos de Adicciones; NATA: Número de artículos de Trastornos de alimentación. *NAT no corresponde con la suma de NAA y NATA ya que un mismo artículo puede tratar tanto de adicciones como de trastornos de la alimentación

Figura 8. Red principal de instituciones (más de 4 colaboraciones)

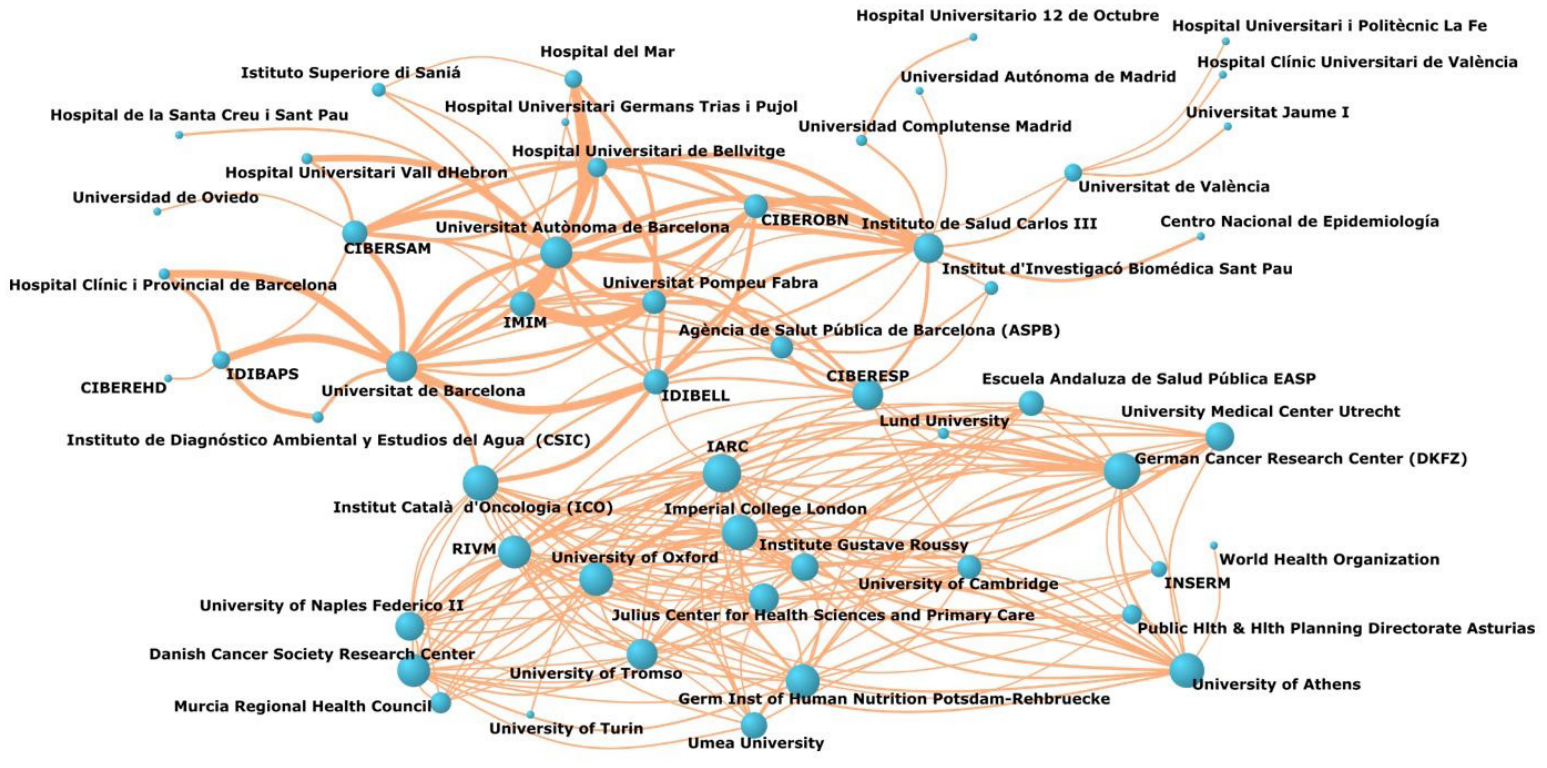


Universitat Autònoma de Barcelona con Centro de Investigación Biomédica en Red de Salud Mental (CIBERSAM) (201). El Instituto de Salud Carlos III colaboró con mayor intensidad con Centro de Investigación Biomédica en Red de la Fisiopatología de la Obesidad y Nutrición (CIBEROBN) (193), Universitat Autònoma de Barcelona (149) y Universitat de Barcelona (142).

\subsection{Colaboración con países extranjeros}

Los países con los que ha colaborado España se presenta en la tabla VII. Estados Unidos es el país con el que se ha colaborado en un mayor número de trabajos ( $n=1.198 ; 7,3 \%)$, seguido de Reino Unido $(n=814,5 \%)$, Italia $(n=727,4,4 \%)$, Alemania $(489,3 \%)$ y Francia $(n=467,2,8 \%)$. Entre las colaboraciones con países latinoamericanos destacan México $(n=169)$, Colombia $(n=109)$, Argentina $(n=107)$, Chile $(n=69)$ y Cuba $(n=53)$, todos ellos excepto México con un porcentaje de trabajos que no llega al $1 \%$. Este ranking se mantiene tanto en el área de las adicciones como en la de los trastornos de la alimentación, donde destaca también la colaboración con Austria $(n=38)$ y Australia $(n=33)$. En número de citas totales en WoS destacan los trabajos con Estados Unidos $(n=34.455)$, Reino Unido (25.432), Italia $(n=24.702)$ y Francia (17.395), mientras que en citas en Scopus el ranking está formado por Estados Unidos, Reino Unido, Italia, Francia y Países Bajos. El mayor número de citas por artículo en WoS corresponde a los trabajos en los que han participado instituciones de China $(C / A=50,09)$, Israel $(C / A=48,34)$ y Japón
$(\mathrm{C} / \mathrm{A}=43,51)$. En el área de las adicciones, en WoS se mantiene el mismo ranking, con la excepción de que las citas de Francia superan a las de Alemania, y en trastornos de la alimentación, Italia supera a Reino Unido y Países Bajos a Francia. En relación con las citas de Scopus, tanto en el área de las adicciones como la de los trastornos de la alimentación, el ranking tiene algunas variaciones: está encabezado por Estados Unidos, seguido de Reino Unido, Italia, Francia, Países Bajos y Alemania.

En la red de colaboración entre países (figura 9) participan 34 países en la que España, que es el país central, muestra un amplio abanico de colaboraciones internacionales, algunas más intensas, como la de Estados Unidos (1131), Reino Unido (769) e Italia (686), y otras menos destacadas con otros países europeos como Alemania (473), Francia (430), Países Bajos (346), Suecia (295), y latinoamericanos (como México, Colombia, Brasil Chile y Argentina, fundamentalmente). También merecen destacarse aquí las colaboraciones con Canadá, Israel, China, Australia, Nueva Zelanda y Japón.

\section{ANÁLISIS TEMÁTICO Y DE ÁMBITOS DEL CONOCIMIENTO}

En la tabla VIII se presentan las áreas temáticas de las revistas en las que se ha publicado un mayor número de artículos junto con las tres palabras clave más frecuentes y las tres revistas con un mayor número de artículos publicados en cada área. Se ha tomado como referencia la asignación temática que establece la Web of Science y las revistas no

Figura 9. Red de colaboración entre países (más de dos colaboraciones)

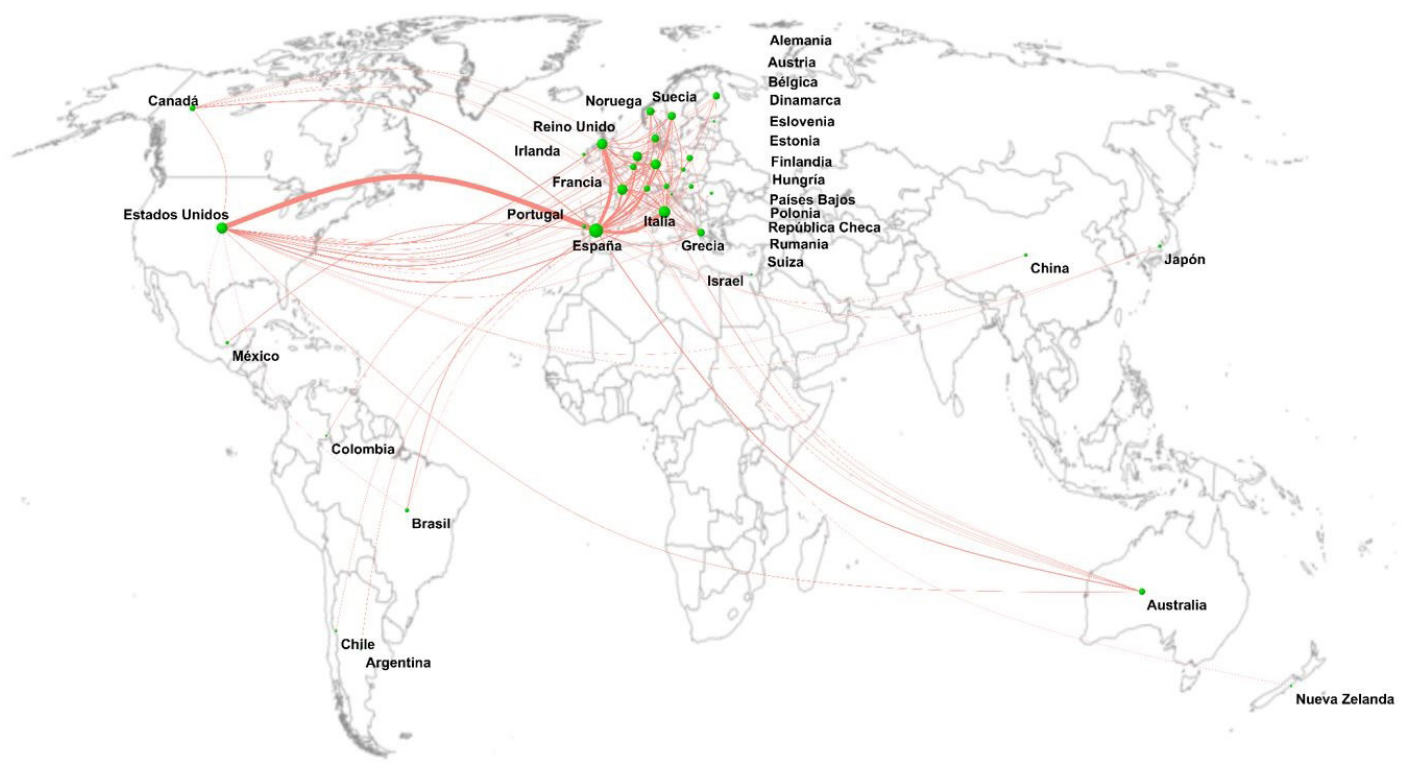


Investigación española en las áreas de adicciones y de trastornos de la alimentación: análisis de la producción, colaboración...

Tabla VII. Países que han colaborado en los trabajos españoles (con 10 o más colaboraciones)

\begin{tabular}{|c|c|c|c|c|c|c|c|c|c|c|c|}
\hline Países & NAT* & NAA & NATA & CW & CW/ A & CW AA & CW ATA & CS & CS / A & CS AA & CS ATA \\
\hline Estados Unidos & 1.198 & 1121 & 127 & 34.455 & 28,76 & 31.614 & 4.192 & 39.266 & 32,78 & 37.173 & 3.767 \\
\hline Reino Unido & 814 & 724 & 114 & 25.432 & 31,24 & 22.817 & 3.154 & 29.159 & 35,82 & 27.020 & 2.772 \\
\hline Italia & 727 & 664 & 86 & 24.702 & 33,98 & 21.867 & 3.357 & 27.038 & 37,19 & 25.478 & 2.167 \\
\hline Alemania & 489 & 451 & 51 & 16.201 & 33,13 & 13.932 & 2.520 & 17.392 & 35,57 & 16.389 & 1.284 \\
\hline Francia & 467 & 442 & 37 & 17.395 & 37,25 & 15.922 & 1.813 & 20.737 & 44,4 & 19.807 & 1.347 \\
\hline Países Bajos & 365 & 342 & 33 & 15.184 & 41,6 & 13.034 & 2.508 & 17.421 & 47,73 & 16.489 & 1.358 \\
\hline Suecia & 312 & 296 & 30 & 12.211 & 39,14 & 11.269 & 1.417 & 12.969 & 41,57 & 12.686 & 828 \\
\hline Suiza & 218 & 205 & 22 & 8.069 & 37,01 & 7.797 & 437 & 8.988 & 41,23 & 8.637 & 554 \\
\hline Australia & 212 & 197 & 33 & 6.470 & 30,52 & 6.243 & 537 & 7.936 & 37,43 & 7.691 & 602 \\
\hline Dinamarca & 204 & 197 & 12 & 7.586 & 37,19 & 7.482 & 274 & 9.044 & 44,33 & 8.899 & 327 \\
\hline Canadá & 201 & 177 & 28 & 6.779 & 33,73 & 5.572 & 1.297 & 5.666 & 28,19 & 5.165 & 602 \\
\hline Portugal & 201 & 194 & 11 & 4.052 & 20,16 & 3.751 & 501 & 4.378 & 21,78 & 4.043 & 563 \\
\hline Bélgica & 191 & 173 & 26 & 6.500 & 34,03 & 5.874 & 913 & 8.317 & 43,54 & 7.596 & 1.076 \\
\hline México & 169 & 155 & 20 & 2.871 & 16,99 & 2.517 & 506 & 3.918 & 23,18 & 3.463 & 626 \\
\hline Brasil & 160 & 146 & 17 & 4.238 & 26,49 & 3.664 & 682 & 4.341 & 27,13 & 3.847 & 637 \\
\hline Grecia & 160 & 157 & 8 & 5.713 & 35,71 & 5.581 & 314 & 6.727 & 42,04 & 6.543 & 396 \\
\hline Noruega & 159 & 151 & 12 & 6.317 & 39,73 & 6.135 & 255 & 7.253 & 45,62 & 7.030 & 311 \\
\hline Finlandia & 129 & 125 & 13 & 4.880 & 37,83 & 4.742 & 210 & 5.311 & 41,17 & 5.150 & 238 \\
\hline Austria & 117 & 89 & 38 & 4.471 & 38,21 & 3.498 & 1.306 & 5.614 & 47,98 & 4.601 & 1.424 \\
\hline Polonia & 111 & 109 & 2 & 4.248 & 38,27 & 3.361 & 887 & 3.851 & 34,69 & 3.740 & 111 \\
\hline Colombia & 109 & 107 & 6 & 2.433 & 22,32 & 2.143 & 419 & 3.453 & 31,68 & 3.133 & 491 \\
\hline Argentina & 107 & 104 & 4 & 2.527 & 23,62 & 2.436 & 92 & 2.934 & 27,42 & 2.922 & 14 \\
\hline Hungría & 77 & 77 & 2 & 2.804 & 36,42 & 2.804 & 69 & 3.119 & 40,51 & 3.119 & 84 \\
\hline Irlanda & 73 & 73 & 2 & 1.774 & 24,3 & 1.774 & 15 & 2.088 & 28,6 & 2.088 & 15 \\
\hline Chile & 69 & 56 & 14 & 1.068 & 15,48 & 937 & 133 & 1.320 & 19,13 & 1.153 & 168 \\
\hline República Checa & 67 & 62 & 7 & 1.815 & 27,09 & 1.662 & 243 & 2.008 & 29,97 & 1.827 & 279 \\
\hline China & 64 & 62 & 4 & 3.206 & 50,09 & 3.199 & 129 & 4.178 & 65,28 & 4.169 & 169 \\
\hline Japón & 61 & 60 & 5 & 2.654 & 43,51 & 2.559 & 226 & 3.349 & 54,9 & 3.238 & 281 \\
\hline Cuba & 53 & 52 & 1 & 918 & 17,32 & 918 & 0 & 1.183 & 22,32 & 1.181 & 2 \\
\hline Rumania & 53 & 51 & 2 & 2.234 & 42,15 & 1.944 & 290 & 2.444 & 46,11 & 2.124 & 320 \\
\hline Israel & 50 & 48 & 4 & 2.417 & 48,34 & 2.401 & 138 & 3.573 & 71,46 & 3.544 & 189 \\
\hline
\end{tabular}

NAT: Número total de artículos; NAA: Número de artículos de Adicciones; NATA: Número de artículos de Trastornos de

alimentación; ARE: número de artículos publicados en revistas extranjeras. ARN: número de artículos publicados en revistas españolas; CW: citas en WoS; CW / A: Citas Wos por artículo; CW AA: Citas WoS Adicciones; CW ATA: Citas WoS Trastornos de la Alimentación; CS: Citas Scopus; CS / A: Citas Scopus por artículo; CS AA: Citas Scopus artículos Adicciones; CS ATA: Citas Scopus Trastornos de la alimentación. *NAT no corresponde con la suma de NAA y NATA ya que un mismo artículo puede tratar tanto de adicciones como de trastornos de la alimentación 


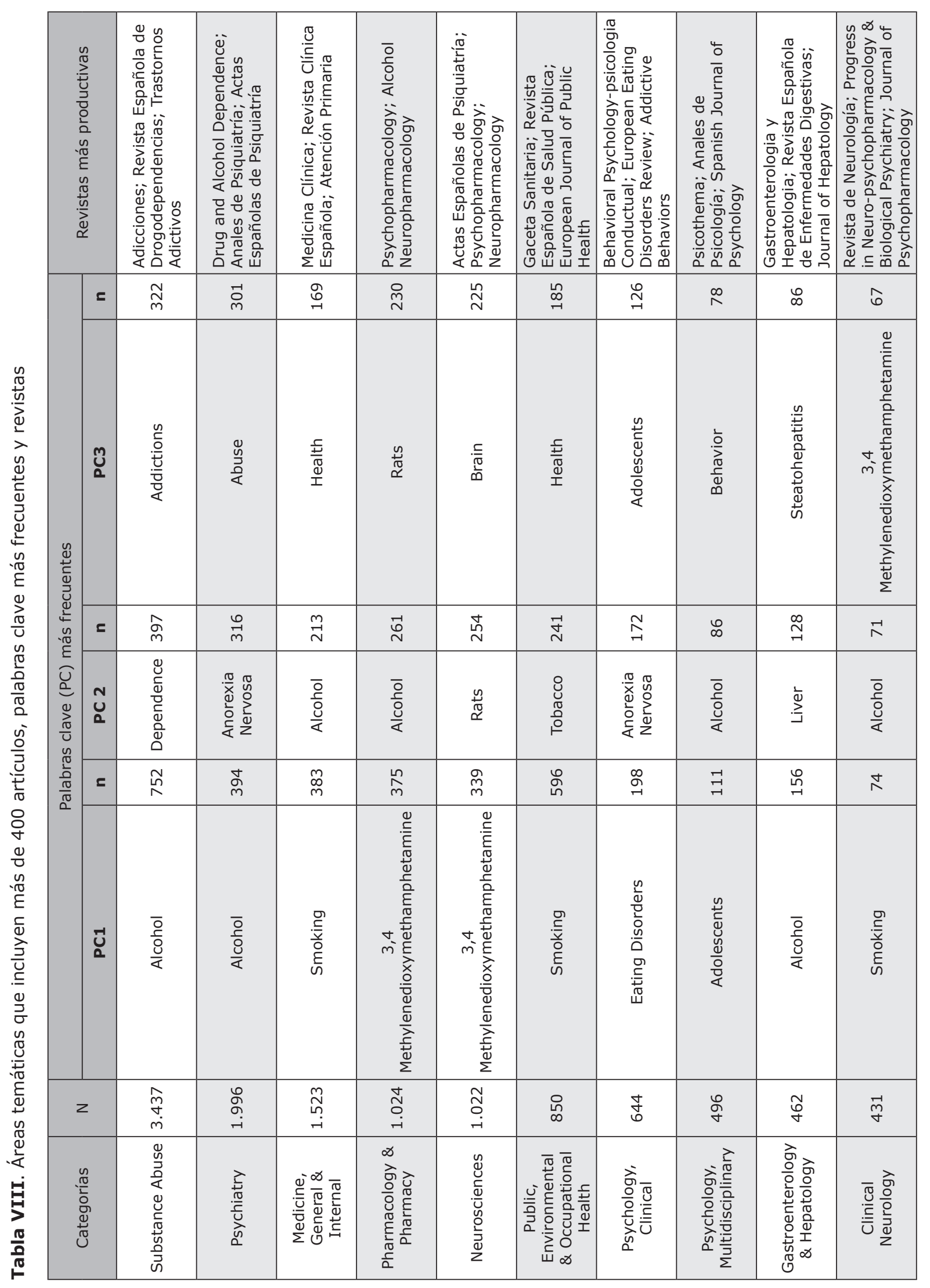


Figura 10. Red de co-ocurrencias de las palabras clave

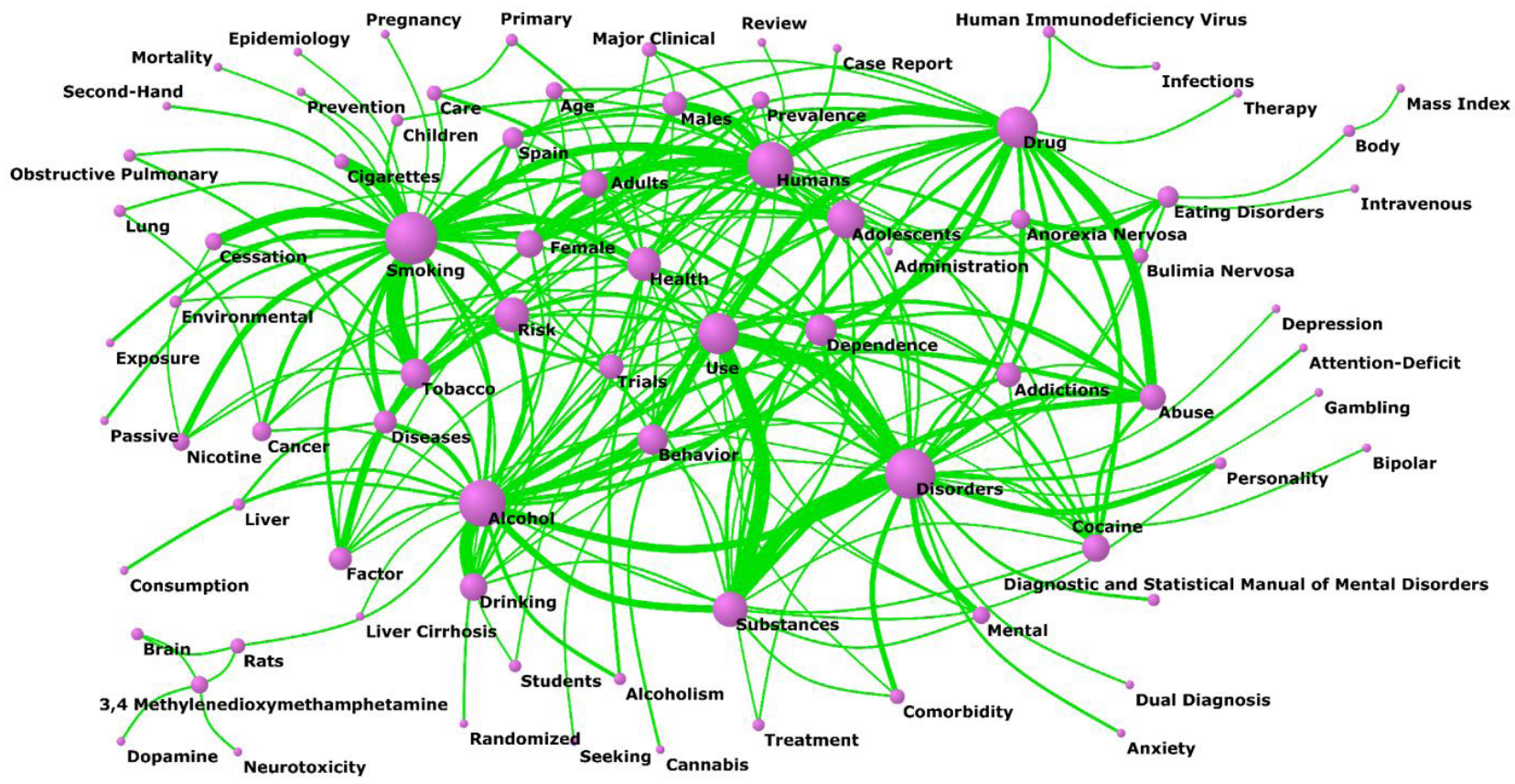

incluidas en esta base de datos se han clasificado manualmente a partir de su título y contenidos. El área que integra un mayor número de trabajos ha sido Substance Abuse $(n=3.437)$ y las tres palabras más frecuentes en esta área han sido "Alcohol" ( $n=752)$, seguida de "Dependence" $(n=397)$ y "Addictions" $(n=322)$. Las tres revistas que han publicado un mayor número de artículos en esta área han sido Adicciones, Revista Española de Drogodependencias y Trastornos Adictivos. La segunda área que incluyó un mayor número de artículos fue Psychiatry $(n=1.996)$, con las palabras clave "Alcohol" $(n=394)$, "Anorexia nervosas" $(n=316)$ y "Abuse" $(n=301)$. En esta área, las revistas con más publicaciones han sido Drug and Alcohol Dependence, Anales de Psiquiatría y Actas Españolas de Psiquiatría. La tercera área más productiva ha sido Medicine, General \& Internal $(n=1.523)$, con las palabras "Smoking" $(\mathrm{n}=383)$, "Alcohol" $(\mathrm{n}=213)$ y "Health" (169) y las revistas Medicina Clínica, Revista Clínica Española y Atención Primaria. Otras áreas que aglutinan numerosos trabajos son Pharmacology \& Pharmacy y Neurosciences, ambas con más de 1.000 artículos, y con más de 500 pero menos de 1.000 artículos dos áreas: Public, Environmental \& Occupational Health y Psychology, Clinical.

El análisis temático se complementa con las redes de co-ocurrencia de palabras clave (palabras clave que coinciden en un mismo artículo). En la red general de co-ocurrencias de palabras clave (fi- gura 10) se ha aplicado un umbral de 180 o más co-ocurrencias en común. En esta red hemos eliminado las palabras de significado vago o genérico que no aportaban información de relevancia, como los tipos de estudio (como "case report"), tipo de trabajo (como "review" y "article") y palabras de significado vago o muy general (como "factor", "primary" e "induced"). La palabra "Smoking" es la que ocupa una posición de mayor centralidad en la red y las mayores co-ocurrencias son "Smoking" con "Tobacco" (1.597) y "Cigarettes" (1.224), y "Alcohol" con "Drinking" (1.232). Un aspecto que destaca es las consecuencias físicas de las adicciones sobre los diversos órganos del ser humano, con términos como "Asthma", "Obstructive pulmonary", "Neurotoxicity" y "Liver cirrosis". Por otra parte, hemos elaborado 6 redes de cowords que hemos aportado como material complementario al artículo para las adicciones al Alcohol, Cannabis, Cocaine, Heroine, Tobacco y Eating disorders. En la red de Cannabis (figura complementaria 3 https://zenodo.org/record/4746351\#.YJlJ5qHtaUk) destacan las palabras "Cocaine", "Alcohol", "Drinking" y "Adolescents"; en la red de Alcohol sobresalen "Behaviour", "Adults", "Adolescents" y "Alcoholism" (figura complementaria 3 https://zenodo. org/record/4746351\#.YJlJ5qHtaUk); en la red de Cocaine destacan "Dependence", "Adults", "Females" y "Alcohol" (figura complementaria 4 https:// zenodo.org/record/4746351\#.YJlJ5qHtaUk); en la red de Heroine predominan "Dependence", "Cocai- 
ne", "Methadone" y "Addictions" (figura complementaria 4 https://zenodo.org/record/4746351\#. YJlJ5qHtaUk); en la red de Tobacco destacan "Smoking", "Cessation", "Nicotine", "Environmental", "Passive" y "Cancer" (figura complementaria 4 https://zenodo.org/record/4746351\#.YJIJ5qHtaUk); Por último, en la red de Eating disorders destacan "Anorexia Nervosa", "Bulimia nervosa"; "Adolescents"; "Body" y "Females" (figura complementaria 5 https://zenodo.org/record/4746351\#. YJIJ5qHtaUk).

\section{DISCUSIÓN}

Este trabajo ha permitido conocer la evolución de la investigación española en el área de las adicciones y en la de los trastornos de la alimentación a lo largo de casi medio siglo, tal y como queda reflejada en las publicaciones científicas. Para obtener las publicaciones españolas de forma exhaustiva, se realizaron búsquedas en base de datos nacionales e internacionales multidisciplinares. Al tratarse de un fenómeno multifactorial y multidisciplinar, para recuperar estas publicaciones fue necesario utilizar perfiles de búsqueda amplios que incluyeran tanto los términos específicos de las conductas adictivas como otros generales relacionados. Se obtuvieron numerosos trabajos que tratan sobre las repercusiones físicas, psíquicas y sociales de estas conductas como, por ejemplo, trabajos sobre cáncer de pulmón en personas fumadoras, o trabajos sobre cirrosis hepática como consecuencia del consumo abusivo del alcohol.

Como se ha podido observar, el número de artículos ha aumentado progresivamente en ambas áreas, sobre todo en la década 2008-2017, periodo en el que se publicó casi la mitad de los trabajos totales. Este crecimiento ha sido observado también en otros trabajos que analizaron algún aspecto de los trastornos adictivos, tanto a nivel nacional (González y otros, 2008), como europeo (Sánchez-Carbonell y otros, 2005) y mundial (Helinski and Spanagel, 2011; Blobaum, 2013; González y otros, 2015; Khalili y otros, 2018; Tran y otros, 2019). A este aumento pueden haber contribuido diversos factores, entre ellos, el crecimiento general de la producción científica producido en muchas áreas en los últimos tiempos, ya sea por el surgimiento de nuevas revistas o por el aumento del número de revistas indizadas en las bases de datos. También puede haber influido el aumento de las partidas destinadas a la financiación de proyectos en el área motivado por las graves consecuencias que tiene el consumo de drogas (Adams, 2007) y el elevado coste del tratamiento de los trastornos y de las enfermedades asociadas a este consumo (Hagemeier, 2018; Rehm, Barbosa, 2018). Una re- visión realizada en 2017 estimó una gran variabilidad en la financiación de estos estudios, que se situaba entre $0,38 €$ y $78 €$ en los proyectos sobre drogas ilícitas, entre $26 €$ y $1.500 €$ euros en los proyectos sobre el consumo de alcohol y entre $10,55 €$ y $391 €$ euros en los de tabaquismo (Barrio y otros, 2017). Debe destacarse que el área de las adicciones cuenta con financiación específica procedente de la Delegación del Gobierno para el Plan Nacional sobre Drogas, que convoca anualmente y desde el año 2000 ayudas a proyectos de investigación a través de las cuales se subvencionan programas prioritarios en el marco del Plan Estatal de Investigación Científica y Técnica y de Innovación. Estas ayudas van dirigidas no sólo a centros de investigación públicos y privados, sino también a organizaciones no gubernamentales, a las que financia programas de formación, prevención, sensibilización y estudio, y a corporaciones locales, a las que se financia programas de prevención del consumo de drogas. A nivel europeo debe destacarse The Justice Programme 2014-2020, que financia proyectos relacionados con las drogas, en particular sobre la coordinación de políticas y la cooperación judicial en cuestiones relacionadas con las drogas y las nuevas sustancias psicoactivas (https://ec.europa.eu/justice/grants1/programmes-2014-2020/ justice/index_en.htm). No se conocen ayudas específicas que financien las investigaciones de los trastornos de la alimentación.

Los trabajos analizados se publicaron en un gran número y variedad de revistas nacionales y extranjeras, tanto específicas de las conductas adictivas como de otras áreas y en otras de propósito general. Sin embargo, el número de artículos en muchas de ellas fue comparativamente bajo, lo que indica una dispersión de las publicaciones y refleja la amplia gama de intereses de investigación relacionados con las adicciones y los trastornos de la alimentación. Este fenómeno también se ha observado en otros trabajos que analizaron las publicaciones sobre adicciones (Zyoud y otros, 2017; Sánchez-Carbonell, 2005; Valderrama-Zurián y otros, 2015). En nuestro trabajo hemos observado que la mayor parte de las revistas más productivas están indexadas en WoS y que el porcentaje de trabajos publicados en revistas situadas en el primer cuartil del JCR ha ido aumentando progresivamente, lo que está en la línea de los datos reportados por otros autores (Moreno Pulido y otros, 2013), que encontraron que el crecimiento medio anual de la producción científica española indizada en WoS era del $9 \%$, mientras que el incremento medio mundial se situaba en el $3 \%$. La revista más productiva del área de trastornos de la alimentación, Europan Eating Disorderes Review, también está clasifica- 
da en el primer cuartil del JCR, pero no ocurre lo mismo con otras revistas españolas como Nutrición Hospitalaria, Actas Españolas de Psiquiatría y Anales de Psiquiatría, ya que se sitúan en los cuartiles tercero y cuarto. Cabe señalar que el factor de impacto medio en JCR del área psicología clínica (edición 2017), en la que se clasifican algunas revistas sobre trastornos de la alimentación, es más bajo que el del área de adicciones (1,952 frente a 2,425$)$. Es importante resaltar la creciente presencia de las revistas españolas en las bases de datos internacionales, ya que se considera que las revistas clasificadas en los cuartiles superiores de las clasificaciones temáticas de las bases de datos que computan las citas recibidas, como WoS y Scopus, son de gran calidad (Glanzel y Moed, 2002; Durieux y Gevenois, 2010; Walters, 2017). Las revistas en las que se publicaron los artículos están clasificadas en numerosas áreas temáticas diferentes, entre las que predominan las relacionas con las ciencias de la salud y la psicología. Esta heterogeneidad es una muestra del carácter interdisciplinario de las dos áreas analizadas, donde profesionales de diferentes especialidades a menudo colaboran para proporcionar investigación científica con una perspectiva suficientemente amplia y multidisciplinar (Granda-Orive y otros, 2009).

De forma paralela al crecimiento de la producción de artículos se ha constatado un notable incremento de los trabajos realizados en colaboración, lo que se explica porque la colaboración es necesaria para el avance del conocimiento pues los problemas requieren abordajes multidisciplinares (Klein, 1996; Bordons y Zulueta, 2002). La colaboración científica permite que los investigadores puedan ampliar su círculo de contactos, fomentar la participación de manera más amplia en los foros de discusión e intercambio de ideas y la ampliación de los grupos y redes de trabajo o la integración en los mismos de nuevos miembros (González-Alcaide y otros, 2008; González de Dios y Aleixandre, 2007; Newman, 2004; Barabásis y otros, 2001). En nuestro trabajo, el índice de colaboración global $(5,19)$ y el encontrado en el área de las adicciones ha sido algo superior al encontrado en estudios previos de temáticas similares, como en el alcoholismo, donde se había incrementado desde el 3,4 autores por trabajo en un estudio a nivel europeo que cubría el periodo $1976-2000$, a 4,8 en otro trabajo durante el periodo 2006-2010 (González-Alcaide y otros, 2013); en otro estudio sobre los trastornos de la conducta alimentaria que analizaba este indicador, el índice fue de 2,4 (Valderrama y otros, 2017), mientras que en nuestro trabajo ha sido de 5,70 . El estudio de la colaboración científica mediante el empleo de técnicas bibliométricas y la identificación de las redes de coautores mediante Análisis de Redes Sociales (ARS) ha permitido identificar los autores que constituyen el frente de investigación en el área y sus colaboraciones con instituciones extranjeras, que en este estudio se produjo en el $21 \%$ de los trabajos. Se ha observado que algunos autores más productivos se incluyen en varias redes simultáneamente, lo que es indicativo de su influencia y de su importancia como un vínculo de conexión entre grupos de investigadores que trabajan en el mismo campo. Un estudio previo encontró una correlación positiva y significativa entre la producción de un autor y su centralidad en las redes, revelando que los autores más productivos eran también más activos en las redes de colaboración (Hou y otros, 2008). Como se ha visto, Estados Unidos es el país con el que se ha colaborado en un mayor número de trabajos en las dos áreas analizadas, seguido de Reino Unido, Italia, Alemania y Francia. Otros países que han destacado, sobre todo en trastornos de la alimentación, han sido Austria, Australia y Países Bajos. El hecho de que la colaboración sea más frecuente con Estados Unidos y con los países europeos se ha observado también en otros estudios (González-Alcaide y otros, 2013; Bramness y otros, 2014) y cabe atribuirlo en gran medida a la concentración de la investigación y el desarrollo científico en estas regiones. Por otra parte, la colaboración con países latinoamericanos es lógica, no sólo por compartir un mismo idioma y por la tradicional hermandad hispanoamericana, sino porque también es resultado de algunas iniciativas llevadas a cabo para implantar programas de investigación en el área que promueven la colaboración internacional (Marín-Navarrete y otros, 2028).

Los trabajos más citados dan una idea de los temas que han despertado más interés o que tienen una metodología más sólida (Tanner-Smith y Polanin, 2016), aunque algunos estudios indican que tasas altas de citación también se asocian a los trabajos que aportan significaciones estadísticas más altas (Jannot y otros, 2013) y a niveles de colaboración más elevados (Figg y otros, 2006). Numerosos trabajos han utilizado anteriormente el análisis de citas en muchas áreas científicas para caracterizar las publicaciones de mayor impacto y conocer los temas que suscitan mayor interés (Bornmann, 2014; Tanner-Smith y and Polanin, 2016; Mahabee-Gittens y otros, 2017). En nuestro estudio, estos documentos tratan sobre adicciones específicas como el tabaquismo y su relación con el cáncer y las enfermedades vasculares, el alcoholismo y sus consecuencias, la MDMA o éxtasis, pero 
también sobre determinados procesos fisiológicos, patológicos y genéticos, sobre todo los mecanismos cerebrales y de recompensa que intervienen en el consumo de drogas y en las recaídas, así como aspectos relacionados con la personalidad y el comportamiento. En general, los artículos de adicciones han recibido más citas que los de trastornos de la alimentación, y de los 18 trabajos más citados, solo dos corresponden a estos últimos.

Del total de trabajos, $14.809(90,52 \%)$ corresponden a adicciones a sustancias, $1.231(7,5 \%)$ a trastornos de la alimentación y en 322 (2\%) trabajos se tratan ambos trastornos en el mismo trabajo. El alcohol y el tabaco son las sustancias que han acaparado la mayor atención de los investigadores a lo largo de todo el periodo estudiado, muy por encima de otras sustancias como el cannabis o la cocaína. En un estudio previo que analizaba más de cincuenta mil artículos sobre adicciones recuperados en varias bases de datos, el $55,2 \%$ de los trabajos analizados estaban relacionados con trastornos por consumo de tabaco y el $38,6 \%$ con trastornos relacionados con el alcohol, mientras que sólo el 4,1\% lo estaban con la cocaína y el 2,6\% con el cannabis (González-Alcaide y otros, 2015). Este porcentaje fue menor en otro estudio limitado a WoS, donde el $32,7 \%$ de los trabajos correspondía al tabaquismo y el 15,4\% al alcoholismo (Tran y otros, 2019).

Un aspecto que llama la atención en los resultados del análisis temático es que mientras que en el caso de del tabaco y el alcohol se identifican numerosos términos sobre enfermedades o trastornos directamente relacionados con su consumo, estas no aparecen en el caso del consumo de otras drogas como opiáceos, cannabis o cocaína. Otro aspecto llamativo es que la frecuencia de las palabras clave relacionadas con el cannabis sea baja, sobre todo por el aumento de consumo detectado en diferentes estudios (es la droga ilegal más consumida tanto en España como en Europa) y el debate abierto hace unos años en torno a su legalización (Hammond y otros, 2020; Grewal y Loh, 2020). La predominancia del tabaco y alcohol están en consonancia con sus tasas de consumo per cápita a nivel mundial. Según la Organización Mundial de la Salud, el consumo total de alcohol per cápita aumentó de 2010 a 2016 (World Health Organization, $\square 2018$ ). Por otra parte, según datos de la XII Encuesta sobre Alcohol y otras Drogas en España 2017-2018 (Delegación del Gobierno para el Plan Nacional sobre Drogas, 2017), el tabaco era la segunda droga más consumida en España y la evolución histórica indicaba que en 2017 se había registrado la mayor prevalencia de los últimos 10 años. También hemos observado la predominancia de algunos términos sobre otros, como el término "female", al que se asocia un mayor número de trabajos que a su homólogo masculino "male". Esto podría ser debido a la creciente preocupación en los últimos años por aspectos relacionados con las diferencias de género y de sexo en diversas áreas de la salud, lo que incluiría al ámbito de las adicciones. Además, llama la atención la predominancia de la palabra clave "adolescence" muy por encima de otros grupos de población como "adults" o "children", lo que parece indicar que la adolescencia es una etapa de la vida que ha generado una clara preocupación en cuanto a producción científica en adicciones y en trastornos de la alimentación. Otro aspecto que llama la atención es la baja presencia de la palabra "prevention", así como otras relacionadas con el ámbito de actuación psicosocial de las adicciones, en detrimento de las que se refieren a conceptos más puramente biomédicos como "brain", "cancer", "liver cirrhosis", "obstructive pulmonary" o "lung".

Este estudio tiene limitaciones que deben considerarse. Una de ellas se debe a la estrategia de búsqueda utilizada y a la selección de las fuentes de información. Aunque los registros se han obtenido de las principales bases de datos nacionales e internacionales, es posible de que se hayan perdido trabajos no indexados en estas fuentes. También es posible que se hayan cometido errores en la normalización de las firmas de los autores e instituciones. Sin embargo, se ha procurado minimizar las consecuencias de todas estas limitaciones mediante un cuidadoso control de los datos haciendo comprobaciones continuas y rigurosas. En cuanto al estudio temático, una tercera parte de los registros no incluían palabras clave, por lo que es posible que el análisis de su frecuencia y co-ocurrencia no refleje exactamente el contenido completo de los artículos de nuestro estudio. Sin embargo, consideramos que el análisis del elevado número de trabajos que sí que las incluyen, ofrecen una aproximación útil al contenido general de estos trabajos. Las citas que han recibido los trabajos publicados en los últimos años deben considerarse con cautela ya que es posible que cuando se hizo la búsqueda no hubiera transcurrido suficiente tiempo como para que los trabajos más recientes fueran citados adecuadamente. Otra limitación relacionada con las citas se refiere al factor de impacto, ya que solamente hemos considerado el indicador en el último año publicado cuando se realizó el estudio (2017) y el factor de impacto de 5 años, y pueden haberse producido cambios importantes en un análisis diacrónico tan amplio como el de este estudio. Sin embargo, por tratarse de un periodo tan amplio y por las dificultades para acceder a los listados por factor de impacto 
de años anteriores, hemos optado por ofrecer el del último año analizado y el de 5 años. Por otra parte, hay que tener en cuenta que si bien el análisis bibliométrico de publicaciones y de su impacto en un campo de investigación científica ofrece indicadores importantes, sería útil relativizarlos con otros sociodemográficos y económicos como, por ejemplo, la población, el producto interior bruto, el número de personas dedicadas a la investigación y los importes destinados a financiar la investigación. Así obtendríamos indicadores relativos que permitirían obtener una idea más completa y precisa de la situación de la investigación sobre las adicciones en España.

En definitiva, nuestro análisis ofrece un panorama amplio de casi medio siglo de investigación sobre adicciones y trastornos de la alimentación en España en comparación con otros análisis bibliométricos que habitualmente comprenden una o dos décadas. Como en anteriores estudios, se ha corroborado un crecimiento del número de trabajos publicados a lo largo del periodo, que ha sido mayor en los últimos quinquenios. Se ha constatado un aumento de las publicaciones en revistas internacionales de gran relevancia, sobre todo en las situadas en los primeros cuartiles del JCR. La visualización de las redes de coautores y de instituciones ha permitido identificar los investigadores y centros más activos en el área. Las redes de co-ocurrencia de palabras han identificado al tabaquismo y al alcoholismo como principales adicciones, pero también otras dependencias con y sin sustancias, y las patologías y consecuencias asociadas. Los resultados de este trabajo pueden servir de apoyo para formular futuras directrices en la investigación.

Futuras líneas de trabajo en este campo podrían analizar la evolución de los indicadores obtenidos en este estudio, tanto de la producción como del impacto científico, así como su asociación con otros indicadores socioeconómicos. También sería relevante seguir la evolución en el tiempo de las redes de coautores y de sus líneas de investigación, así como detectar el nacimiento de nuevas redes, el surgimiento de nuevas líneas de estudio o de adicciones emergentes. Otro aspecto relevante es el análisis de la especialización de las redes de investigación en determinadas temáticas, así como su grado de colaboración con redes internacionales. Otro campo que convendría analizar es el relativo a la transferencia de conocimiento a la sociedad y su divulgación al gran público en los medios de comunicación. Por último, sería interesante analizar el impacto que tienen las ayudas que las entidades financiadoras destinan a la investigación en este campo.

\section{AGRADECIMIENTOS}

Delegación de Gobierno para el Plan Nacional sobre Drogas (convocatoria 2016. Proyecto 2016/028). Ministerio de Sanidad Consumo y Bienestar Social.

Servicio de Drogodependencias (PMD/UPCCA-València). Concejalía de Sanidad y Consumo. Ajuntament de València.

\section{ACKNOWLEDGEMENTS}

Government Delegation for the National Plan on Drugs (call 2016. Project 2016/028). Ministry of Health, Consumption and Social Welfare.

Drug Addiction Service (PMD / UPCCA-València). Department of Health and Consumption. Valencia City Council.

\section{REFERENCIAS}

Adams, P.J. (2007). Assessing whether to receive funding support from tobacco, alcohol, gambling and other dangerous consumption industries. Addiction, 102(7), 1027-1033. https://doi.org/10.1111/j.13600443.2007.01829.x

Akbar, H.F.; Siddiq, K.; Nusrat, S. (2019). Citation Classics and Trends in the Field of opioids: A Bibliometric Analysis. Cureus, 11(7). doi:10.7759/cureus.5055

Álvarez, F.J..; Aleixandre-Benavent, R. (2001). Bases documentales en drogodependencias. En: F. Giner y G. Cervera, Trastornos Adictivos. Drogodependencias: clínica y tratamientos psicológicos, pp. 85-113. Valencia: Generalitat Valenciana. Conselleria de Benestar Social.

APA. (2013). Diagnostic and Statistical Manual of Mental Disorders (DSM-V) (Fifth Edition). Washington, DC: American Psychiatric Association.

APA. (2000). Diagnostic and Statistical Manual of Mental Disorders (DSM-IV-TR). Washington, DC: American Psychiatric Association.

Arbinaga Ibarzábal, F. (2002). Análisis bibliométrico de la revista "Adicciones" (1996-2000). Adicciones, 14 (2). https://doi.org/10.20882/adicciones.495

Bahji, A.; Mazhar, M.N.; Hudson, C.C.; Nadkarni, P.; MacNeil, B.; Hawken, E. (2019). Prevalence of substance use disorder comorbidity among individuals with eating disorders: A systematic review and meta-analysis. Psychiatry Research.; 273, 58-66. https://doi. org/10.1016/j.psychres.2019.01.007

Barabásis, A.L.; Jeong, H.; Néda, Z.; Ravasz, E.; Dchubert, A.; Vicsek, T. (2001). Evolution of the social network of scientific collaborations. Physica A: Statistical Mechanics and its Applications, 311(3-4), 594-610. https://doi.org/10.1016/S0378-4371(02)00736-7

Barrio, P.; Reynolds, J.; García-Altés, A. ; Gual, A. ; Anderson, P. (2017). Social costs of illegal drugs, alcohol and tobacco in the European Union: A systematic review. Drug and Alcohol Review, 36(5), 578-588. https://doi. org/10.1111/dar.12504

Batman, A.M.; Miles, M.F. (2015). Translating Alcohol Research: Opportunities and Challenges. Alcohol Research, 37(1), 7-14 
Blobaum P.M. (2013). Mapping the literature of addictions treatment. Journal of the Medical Library Association, 101(2), 101-9. https://doi.org/10.3163/15365050.101.2.005

Bordons, M.; Zulueta, M.A. (2002). La interdisciplinariedad en los grupos españoles de investigación en el área cardiovascular. Revista Española de Cardiología, 55, 900-912. https://doi.org/10.1016/S03008932(02)76728-6

Bornmann, L. (2014). How are excellent (highly cited) papers defined in bibliometrics? A quantitative analysis of the literature. Research Evaluation, 23, 166-173. https://doi.org/10.1093/reseval/rvu002

Bramness, J.G.; Henriksen, B.; Person, O.; Mann, K. (2014) A bibliometric analysis of European versus USA research in the field of addiction. Research on alcohol, narcotics, prescription drug abuse, tobacco and steroids 2001-2011. European Addiction Research, 20(1), 16-22. https://doi.org/10.1159/000348260

Briganti, M.; Delnevo, C.D.; Brown, L.; Hastings, S.E.; Steinberg, M.B. (2019). Bibliometric Analysis of Electronic Cigarette Publications: 2003 $\square$ 2018. International Journal of Environmental Research and Public Health, 16 (3). https://doi.org/10.3390/ijerph16030320

Carbonell, X. (2014). La adicción a los videojuegos en el DSM-5. Adicciones, 26 (2), 91-95. https://doi. org/10.20882/adicciones. 10

Carta, M.G.; Preti, A.; Moro, M.F.; Aguglia, E.; Balestrieri, M.; Caraci, F, Dell'Osso, L. ; Di Sciascio, G.; Drago, F.; Faravelli, C. ; Hardoy, M.C.; D’Aloja, E. ; Cossu, G. ; Calò, S. ; Palumbo, G.; Bhugra, D. (2014). Eating disorders as a public health issue: prevalence and attributable impairment of quality of life in an Italian community sample. International Review of Psychiatry, 26(4), 486-92. https://doi.org/10.3109/09540261.2014.927753

Cliffor, A.; Shakeshaft, A. (2017). A bibliometric review of drug and alcohol research focused on Indigenous peoples of Australia, New Zealand, Canada and the United States. Drug and Alcohol Review, 36(4), 509-522. https://doi.org/10.1111/dar. 12510

De Granda Orive J.I.; Aleixandre Benavent, R.; García Río, F.; Solano, S.; Villanueva, S.; Alonso Arroyo, A.; Valderrama, J.C. (2009). World-wide collaboration among medical specialties in smoking research: Production, collaboration, visibility and influence. Research Evaluation, 18(1), 3-12. https://doi.or$\mathrm{g} / 10.3152 / 095820209 \times 393163$

De Miguel, A. (1994). La sociedad española 1993-94. Madrid: Alianza.

Delegación del Gobierno para el Plan Nacional sobre Drogas. EDADES (2017). Encuesta sobre alcohol y otras drogas en España (EDADES), 1995-2017. Madrid: Delegación del Gobierno para el Plan Nacional sobre Drogas, 2017.

Dorta-González, P.; Dorta-González, M.I. (2013). Comparing journals from different fields of science and social science through a JCR subject categories normalized impact factor. Scientometrics, 95, 645-72. https://doi.org/10.1007/s11192-012-0929-9

Durieux, V.; Gevenois, P.A. (2010). Bibliometric Indicators: Quality Measurements of Scientific Publication. Radiology, 255(2), 342-351. https://doi.org/10.1148/ radiol.09090626
EMCDDA. (s.f). EMCDDA, your reference point on drugs in Europe. Disponible en: http://www.emcdda.europa. eu/about [Fecha de consulta: 02/03/2020].

Figg, W.D. ; Dunn, L.; Liewehr, D.J.; Birkinshaw, J. (2006). Scientific collaboration results in higher citation rates of published articles. Pharmacotherapy, 26, 759-767. https://doi.org/10.1592/phco.26.6.759

Glanzel, W.; Moed, H.F. (2002). Journal impact measures in bibliometric research. Scientometrics, 53 (2), 171193. https://doi.org/10.1023/A:1014848323806

González-Alcaide, G.; Castelló-Cogollos, L.; Castellano-Gómez, M.; Agullo-Calatayud, V.; Aleixandre-Benavent, R.; Alvarez, F.J.; Valderrama-Zurián, J.C. (2013). Scientific publications and research groups on alcohol consumption and related problems worldwide: authorship analysis of papers indexed in PubMed and Scopus databases (2005 to 2009). Alcoholism: Clinical and Experimental Research, 37, 381-93. https:// doi.org/10.1111/j.1530-0277.2012.01934.x

González-Alcaide, G.; Bolaños-Pizarro, M.; Navarro-Molina, C.: De Granda Orive J.I.; Aleixandre-Benavent, R. ; Valderrama-Zurián, J.C. (2008). Análisis de la producción científica española sobre abuso de sustancias en función de la colaboración entre disciplinas. Adicciones, 20, 337. doi:10.20882/adicciones.737. https:// doi.org/10.20882/adicciones. 737

González-Alcaide, G.; Fernandes, L.; Pinto, M.; Agulló Calatayud V. (2015). La investigación biomédica sobre abuso de sustancias a través de la base de datos Medline (2006-2010). Revista Española de Drogodependencias, $40,29-45$.

González de Dios, J.; Aleixandre, R. (2007). Evaluación de la investigación en Biomedicina y Ciencias de la Salud. Indicadores bibliométricos y cienciométricos. Boletín de Pediatría, 47, 92-110.

Grewal, J.K.; Loh, L.C. (2020). Health considerations of the legalization of cannabis edibles. Canadian Medical Association Journal, 192(1), E1-E2. https://doi. org/10.1503/cmaj.191217

Hagemeier, N.E. (2018). Introduction to the opioid epidemic: the economic burden on the healthcare system and impact on quality of life. American Journal of Management Care, 24(10 Suppl), S200-S206.

Hammond, D.; Goodman, S.; Wadsworth, E.; Rynard, V.; Boudreau, C.; Hall, W. (2020). Evaluating the impacts of cannabis legalization: The International Cannabis Policy Study. International Journal of Drug Policy, 77, 102698. https://doi.org/10.1016/j.drugpo.2020.102698

Helinski, S.; Spanagel, R. (2011). Publication trends in addiction research. Addiction Biology, 16(4), 532-9. https://doi.org/10.1111/j.1369-1600.2011.00388.x

Hou, H.; H Kretschmer, H and Liu, Z. (2008). The structure of scientific collaboration networks in Scientometrics. Scientometrics, 75, 189-202. https://doi. org/10.1007/s11192-007-1771-3

Hudson, J.I.; Pope, H.G. (2018). Evolving Perspectives on the Public Health Burden of Eating Disorders. Biological Psychiatry, 84(5), 318-319. https://doi.org/10.1016/j. biopsych.2018.06.011

Jannot, A.S.; Agoritsas, T.; Gayet-Ageron, A.; Perneger, T.V. (2013). Citation bias favoring statistically signifi- 
cant studies was present in medical research. Journal of Clinical Epidemiology, 66: 296-301. https://doi.org/10.1016/j.jclinepi.2012.09.015

Khalili, M.; Rahimi-Movaghar, A.; Shadloo, B.; Mojtabai, R.; Mann, K.; Amin-Esmaeili, M. (2018), Global Scientific Production on Illicit Drug Addiction: A Two-Decade Analysis. European Addiction Research, 24(2), 60-70. https://doi.org/10.1159/000487590

Klein, J.T. (2004). Interdisciplinarity and complexity: an evolving relationship. EconoJournal, 6, 2-10.

Kushner, M.G. (2014). Seventy-five years of comorbidity research. Journal of Studies on Alcohol and Drugs, 17, 50-58. https://doi.org/10.15288/jsads.2014.s17.50

Leydesdorff, L.; Bornmann, L. (2011). How fractional counting of citations affects the impact factor: Normalization in terms of differences in citation potentials among fields of science. Journal of the Association for Information Science and Technology, 62, 217-229. https://doi.org/10.1002/asi.21450

Li, D.; Okamoto, J.; Liu, H.; Leischow, S. (2015). A bibliometric analysis on tobacco regulation investigators. BioData Mining, 8, 11. https://doi.org/10.1186/ s13040-015-0043-7

Mahabee-Gittens, E.M.; Gordon, J.S.; Melink, K.F.; Merianos, A.L. (2017). Top 100 Cited Articles in Recent Tobacco Research. Journal of Behavioral Health, 6(1), 16-25. https://doi.org/10.5455/jbh.20160814123529

Marín-Navarrete, R.; Medina-Mora, M.E.; Pérez-López, A.; Horigian, V.E. (2018). Development and evaluation of addiction treatment programs in Latin America. Current Opinion in Psychiatry, 31(4), 306-314. https:// doi.org/10.1097/YCO.0000000000000434

Metse, A.P.; Wiggers, J.H. ; Wye, P.M.; Wolfenden, L. ; Prochaska, J.J.; Stockings, E.A.; Williams, J.M.; Ansell, K.; Fehily, C.; Bowman, J.A. (2017). Smoking and Mental Illness: A Bibliometric Analysis of Research Output Over Time. Nicotine and Tobacco Research, 19(1), 2431. https://doi.org/10.1093/ntr/ntw249

Moessner, M.; Bauer, S. (2017). Maximizing the public health impact of eating disorder services: A simulation study. International Journal of Eating Disorders, 50(12), 1378-1384. https://doi.org/10.1002/eat.22792

Mond, J.M. (2016). Optimizing prevention programs and maximizing public health impact are not the same thing. Eating Disorders, 24(1), 20-8. https://doi.org/ $10.1080 / 10640266.2015 .1113824$

Moreira, M.R.; Fernandes, F.M.; Ribeiro, J.M.; Franco Neto, Tde L. (2015). A review of Brazilian scientific output on crack--contributions to the political agenda. Revista Ciência \& Saúde Coletiva, 20(4), 1047-62. https://doi.org/10.1590/1413-81232015204.03202014

Moreno-Pulido, A.; López-González, M.A.; Rubio-Garay, F.; Saúl, L.A.; Sánchez-Elvira-Paniagua, A. (2013). Evolución de las revistas españolas de Ciencias Sociales en el Journal Citation Reports (2006-2010) y su valoración como indicio de calidad en la normativa evaluadora española. Revista Española de Documentación Científica, 36 (3), e014. https://doi.org/10.3989/redc.2013.3.987 https://doi.org/10.3989/redc.2013.3.987

National Institute on Drug Abuse. (s.f). About NIDA. Disponible en: https://www.drugabuse.gov/about-nida [Fecha de consulta: 02/03/2020].
Neff, J.A.; Gunsolley, J.C.; Alshatrat, S.M. Topical trends in tobacco and alcohol articles published in three dental journals, 1980-2010. Journal of Dental Education, 79(6), 671-9. https://doi.org/10.1002/j.00220337.2015.79.6.tb05940.x

Newman, M.E. (2004). Coauthorship networks and patterns of scientific collaboration. Proceedings of the National Academy of Sciences, 101, 5200-5. https://doi. org/10.1073/pnas.0307545100

National Institute on Drug Abuse. (s.f). About NIDA. Disponible en: https://www.drugabuse.gov/about-nida [Fecha de consulta: 02/03/2020].

Observatorio Español de las Drogas y las Adicciones. Delegación del Gobierno para el Plan Nacional sobre Drogas. (2019). Informe sobre adicciones comportamentales. Juego y uso compulsivo de internet en las encuestas de drogas y adicciones en España EDADES y ESTUDES. Madrid: Ministerio de Sanidad, Consumo y Bienestar Social.

PNSD. (1997). Prevención de las drogodependencias. Análisis y propuestas de actuación. Madrid: Delegación del Gobierno para el Plan Nacional sobre Drogas.

Rehm, J.; Barbosa C. (2018). The cost-effectiveness of therapies to treat alcohol use disorders. Expert Review of Pharmacoeconomics Outcomes Research, 18(1), 43-49. https://doi.org/10.1080/14737167.2018.1392 241

Rizkallah, J.; Sin, D.D. (2019). Integrative approach to quality assessment of medical journals using impact factor, eigenfactor, and article influence scores. PloS One, 5, e10204. DOI: 10.1371/journal.pone.0010204. https://doi.org/10.1371/journal.pone.0010204

Sánchez-Carbonell, X.; Guardiola, E.; Bellés, A.; Beranuy, M. (2005). European Union scientific production on alcohol and drug misuse (1976-2000). Addiction, 100, 1166-1174 https://doi.org/10.1111/j.13600443.2005.01135.x

Tanner-Smith, E.E.; Polanin, J.R. (2016). Brief alcohol intervention trials conducted by higher prestige authors and published in higher impact factor journals are cited more frequently. Journal of Clinical Epidemiology, 75, 119-25. https://doi.org/10.1016/j.jclinepi.2016.01.028

Torres Hernández, M.A.; Santo Domingo Carrasco, J.; Pascual Pastor, F.; Freixa Santfeliu, F.; Álvarez Vara, C. (2008). Historia de las adicciones en la España contemporánea. Madrid: Delegación del Gobierno para el Plan Nacional sobre Drogas.

Tran, B.X.; Moir, M.; Latkin, C.A.; Hall, B.J.; Nguyen, C.T.; Ha, G.H, Nguyen, Nam Ba.; Cyrus, S.Ho.; Roger, C.M.Ho. (2019). Global research mapping of substance use disorder and treatment 1971-2017: implications for priority setting. Substance Abuse Treatment Prevention and Policy,14(1), 21. https://doi.org/10.1186/ s13011-019-0204-7

Valderrama Zurián, J.C. ; Aleixandre Benavent, R.; Gisbert Tío, A. (1997). Repertorios bibliográficos y bases de datos en ciencias de la salud de interés en drogodependencias. Revista Española de Drogodependencias, 22(2), 125-34.

Valderrama-Zurián, J.C. (2000). Estudio bibliométrico de las publicaciones españolas sobre drogodependencias 
en Medicina (1989-1994). (tesis doctoral). València: Universitat de València,

Valderrama-Zurián, JC.; Melero-Fuentes, D.; Aleixandre-Benavent, R. (2015). Bibliographic searches for a bibliometric analysis on drug addiction. European Addiction Research, 21(1), 31-2. https://doi. org/10.1159/000365081

Valderrama-Zurián, J.C.; Aguilar-Moya, R.; Cepeda-Benito, A.; Melero-Fuentes, D.; Navarro-Moreno, M.A.; Gandía-Balaguer, A.; Aleixandre-Benavent, R. (2017). Productivity trends and collaboration patterns: A diachronic study in the eating disorders field. PLoS ONE, 12(8). https://doi.org/10.1371/journal. pone. 0182760

Villanueva Serrano, S.; De Granda Orive, J.I.; Aleixandre Benavent, R.; García Río, F.; Valderrama Zurián, J.C, Alonso Arroyo, A. (2007). Análisis de la red de colaboración científica sobre tabaquismo entre centros sanitarios españoles a través del Science Citation Index (1999 - 2003). Archivos de Bronconeumología, 43(7), 378-385 https://doi.org/10.1157/13107694

Walters, W.H. (2017). Citation-Based Journal Rankings: Key Questions, Metrics, and Data Sources. IEEE Access, 5. 22036 - 22053. DOI:10.1109/ACCESS.2017.2761400 https://doi.org/10.1109/AC-
Wang, J.H.; Wang, M.; Liu, S.C.; Du, X.F.; Han, M.; Liu, J.F.; y otros A bibliometric analysis of clinical study literature of traditional Chinese medicine therapies for smoking cessation. Tobacco Induced Diseases, 16:15. https://doi.org/10.18332/tid/86330

Willemsen, M.C.; Nagelhout, G.E. (2016). Country Differences and Changes in Focus of Scientific Tobacco Control Publications between 2000 and 2012 in Europe. European Addiction Research, 22(1), 52-8. https:// doi.org/10.1159/000381674

World Health Organization (2018). Global status report on alcohol and health 2018. World Health Organization. Disponible en: https://apps.who.int/iris/handle/10665/274603. [Fecha de consulta: 02/03/2020]

Zyoud, S.H.; Waring, W.S.; Al-Jabi, S.W.; Sweileh, W.M. (2017). Global cocaine intoxication research trends during 1975-2015: a bibliometric analysis of Web of Science publications. Substance Abuse Treatment Prevention and Policy, 12(1), 6. https://doi.org/10.1186/ s13011-017-0090-9

Zyoud, S.H. (2018). Estimates of global research productivity in using nicotine replacement therapy for tobacco cessation: a bibliometric study. Global Health, 14(1), 14. https://doi.org/10.1186/s12992-018-0335-z 